\title{
Spatial distribution of atypical aftershocks of the 1995 Hyogo-ken Nanbu earthquake
}

\author{
Hiroshi Yamanaka ${ }^{1}$, Yoshihiro Hiramatsu ${ }^{1}$, and Hiroshi Katao ${ }^{2}$ \\ ${ }^{1}$ Department of Earth Sciences, Faculty of Science, Kanazawa University, Ishikawa 920-1192, Japan \\ ${ }^{2}$ Research Center for Earthquake Prediction, Disaster Prevention Research Institute, Kyoto University, Kyoto 611-0011, Japan
}

(Received December 21, 2000; Revised September 24, 2002; Accepted September 26, 2002)

The 1995 Hyogo-ken Nanbu earthquake (MJMA 7.3) occurred on January 17, 1995. To reveal the detailed stress field after the mainshock, we relocate hypocenters of aftershocks $(M \geq 2.3)$ and determine their focal mechanisms using seismic records obtained by GROUPS-95, a temporary dense seismic network in and around the aftershock region. Along the mainshock rupture zone, some aftershocks are nearly N-S compression or normal fault type events, which is inconsistent with the regional stress field of approximately horizontal E-W compression. We call these aftershocks atypical, defined as events which have focal mechanisms with P-axis directions more than $45^{\circ}$ from the regional stress field. The atypical aftershocks amount to about $17 \%$ of the total. No temporal variations in aftershock mechanism are found in the analysis period. Their spatial distribution is compared with the slip and stress distribution of the mainshock, as well as the P- and S-wave velocity structure. Most of them are located at the upper boundary between the slipped and unslipped zones of the mainshock. We suggest that the atypical aftershocks are caused by the disturbance of the local tectonic stress field due to the heterogeneous coseismic slip.

\section{Introduction}

The 1995 Hyogo-ken Nanbu earthquake occurred on January 17,1995 with a magnitude of 7.3 determined by the Japan Meteorological Agency. The hypocenter of the mainshock was located beneath the Akashi Strait in the Kinki District, Japan (Fig. 1). There was severe damage in a wide area, including Kobe city, and more than 6,000 people were killed.

Many aftershocks occurred on the Rokko Fault System, located on the northeastern side of the Akashi Strait, and in the northeastern part of Awaji Island located on the southwestern side of the Akashi Strait, as shown in Fig. 2. The aftershock region extends about $70 \mathrm{~km}$ from northeast to southwest (Hirata et al., 1996; Katao et al., 1997). The surface rupture caused by the mainshock was observed along the Nojima Fault which is located on the northwestern coast of Awaji Island (Nakata et al., 1995; Awata et al., 1996). The observation of microearthquake activity for the past 30 years clearly shows that the seismicity along the mainshock rupture zone was low on the southern side of the Arima-Takatsuki Tectonic Line (ATTL) before the mainshock, while the seismicity has been high in the Tamba Plateau on the northern side of ATTL (Katao et al., 1997; Hiramatsu et al., 2000).

The 1995 Hyogo-ken Nanbu earthquake presents many seismologically interesting aspects, including the feature of the aftershock distribution (Hirata et al., 1996; Nakamura and Ando, 1996; Katao et al., 1997), the discrepancy in the fault configuration beneath Akashi Strait (Nakamura and Ando, 1996; Takahashi et al., 1996), the regional stress field after the mainshock (Katao et al., 1997), the slip distribution

Copy right (C) The Society of Geomagnetism and Earth, Planetary and Space Sciences (SGEPSS); The Seismological Society of Japan; The Volcanological Society of Japan; The Geodetic Society of Japan; The Japanese Society for Planetary Sciences. of the mainshock (Bouchon et al., 1998; Ide and Takeo, 1997), the stress distribution on the rupture fault before and after the mainshock (Bouchon et al., 1998), the P- and Swave velocity structure (Zhao and Negishi, 1998), and the $\mathrm{S}$-wave splitting in the aftershock region (Tadokoro et al., 1999).

Comparisons of aftershock distribution with coseismic slip distribution have been done for numerous earthquakes. Mendoza and Hartzell (1988) showed that aftershocks following a large earthquake occur mostly outside of or near the edges of the area of the maximum slip (e.g. 1986 North Palm Springs earthquake, 1983 Borah Peak earthquake). They suggested that slips are continued the outer region of the mainshock rupture area or subsidiary faults are activated.

To investigate the stress field after the Hyogo-ken Nanbu earthquake in and around the mainshock rupture zone, Katao et al. (1997) determined the focal mechanisms of aftershocks using permanent seismic networks, and compared them with those of the microearthquakes before the mainshock reported by Iio (1996). Katao et al. (1997) reported that some aftershocks showed nearly horizontal N-S or vertical compression axes. Their mechanisms were not consistent with the regional stress field of approximately horizontal E-W compression. Because the stations of the permanent seismic networks were not distributed uniformly over the mainshock rupture, the accuracy of these mechanisms determined was limited.

The Geophysical Research Group Organized by the Universities for Prediction Seismology in 1995 (GROUPS-95) installed 27 temporary seismic stations in and around the aftershock region a week after the mainshock (Hirata et al., 1996) (Fig. 3). Using the seismic records obtained by GROUPS-95, we relocate aftershock hypocenters $(\mathrm{M} \geq 2.3)$, 


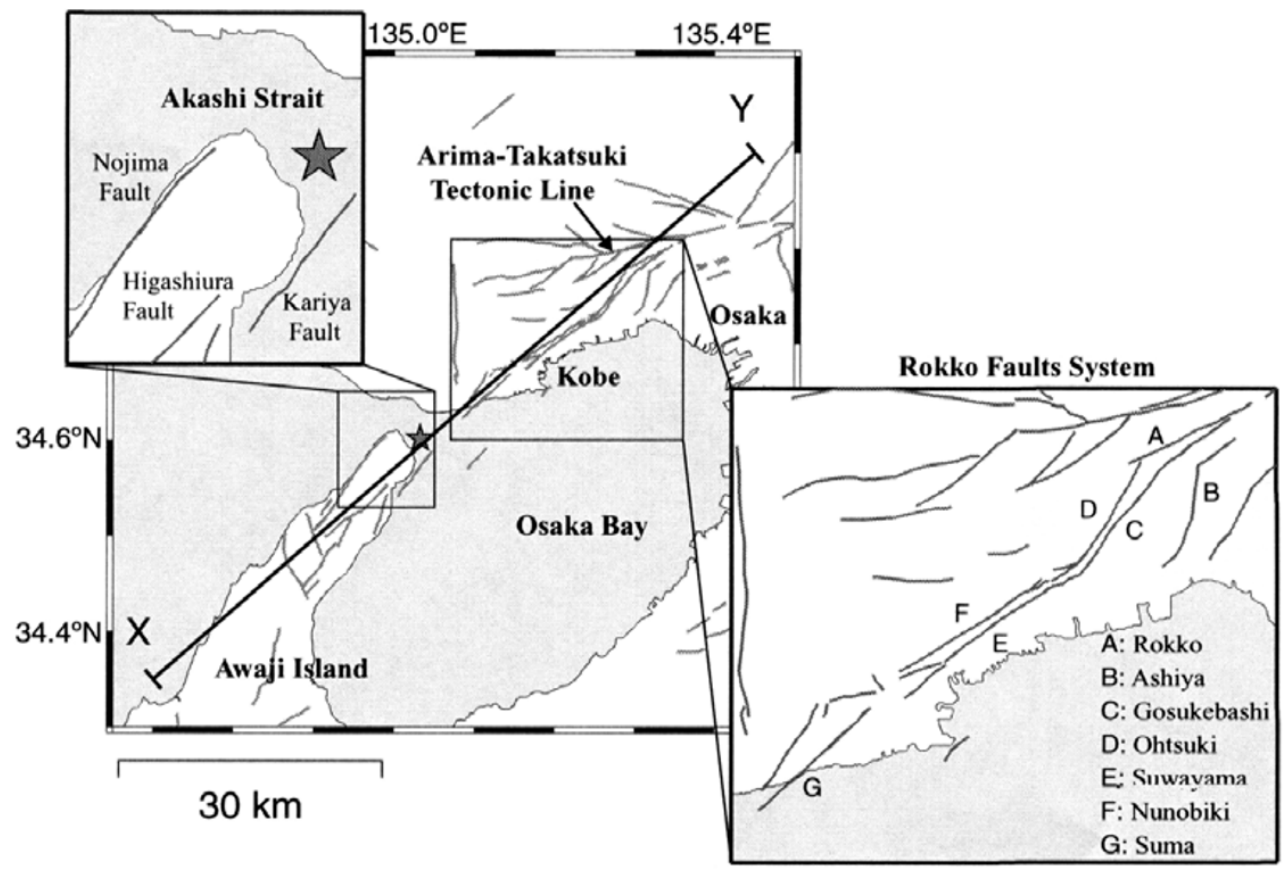

Fig. 1. Distribution of active faults. The Rokko Fault System consists of seven major active faults; (A) Rokko Fault, (B) Ashiya Fault, (C) Gosukebashi Fault, (D) Ohtsuki Fault, (E) Suwayama Fault, (F) Nunobiki Fault and (G) Suma Fault. Line X-Y shows the orientation of the cross section in Figs. 8-11. Star shows the epicenter of the mainshock.

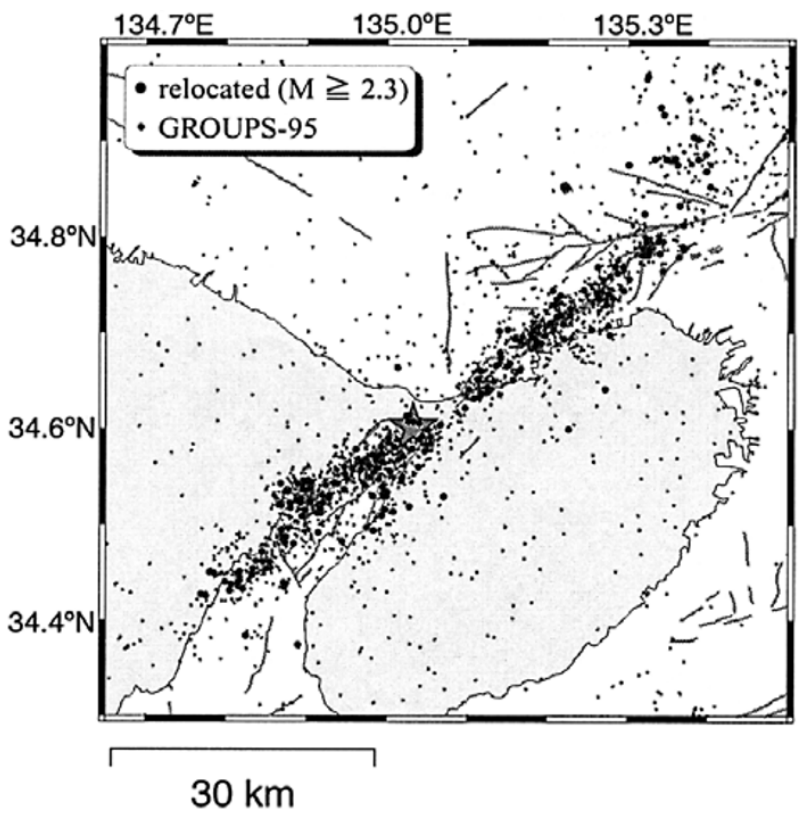

Fig. 2. Distribution of the aftershocks of the 1995 Hyogo-ken Nanbu earthquake. Small dots show the original aftershock locations determined by the GROUPS-95, while solid circles show those $(\mathrm{M} \geq 2.3)$ relocated in this study.

and determine their focal mechanisms using P-wave initials. This dense seismic network enables us to determine the locations and focal mechanisms of the aftershocks with a high accuracy. We can also infer the post-seismic stress distribution along the mainshock rupture from these focal mechanisms.

A primary objective of this paper is to describe the characteristics of the spatial distribution of the aftershocks and their focal mechanisms. We compare our results with vari-

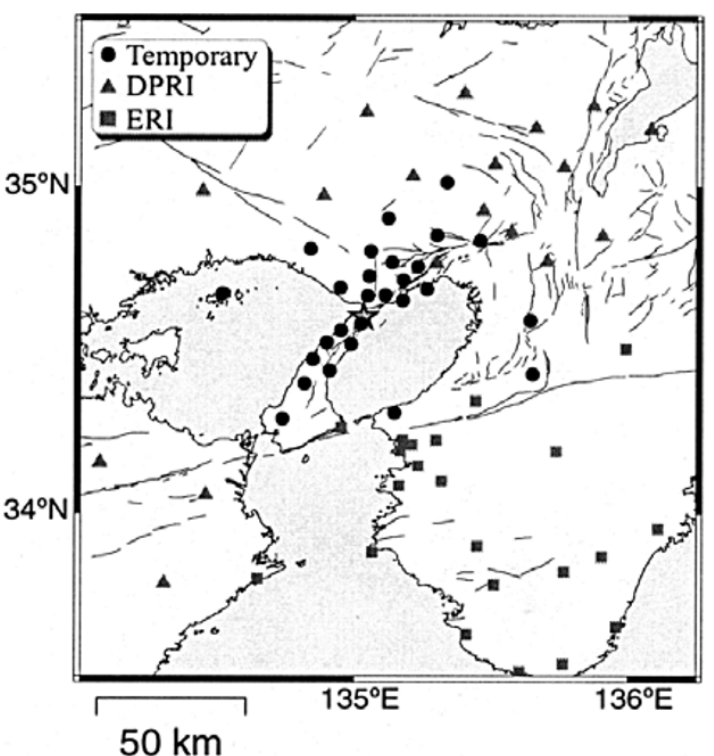

Fig. 3. Distribution of the seismic stations used in this study. Solid circles are the temporary stations deployed by GROUPS-95 after the Hyogo-ken Nanbu earthquake. Gray symbols represent the permanent seismic networks: triangles are the Abuyama, Tottori and Tokushima networks operated by DPRI, Kyoto University, and squares are the Wakayama network operated by ERI, the University of Tokyo. Star shows the epicenter of the mainshock.

ous other geophysical results for the 1995 Hyogo-ken Nanbu earthquake, such as slip and stress distributions and P- and $\mathrm{S}$-wave velocity structures. We show that most of the aftershocks occurred in areas of high stress after the mainshock, while atypical aftershocks, defined later in the discussion section, mainly occurred at the upper boundary of the areas of the maximum coseismic displacement. 
Table 1. Focal mechanism solutions of the aftershocks $(\mathrm{M}>2.3)$ in the 1st observation period: Time is Japanese Standard Time. The fault parameters follow the notations defined by Aki and Richards (1980). (i.e., Plunge of P-axis and T-axis are measured down from the horizontal.)

\begin{tabular}{|c|c|c|c|c|c|c|c|c|c|c|c|c|c|c|c|c|}
\hline & & & & & & & & & & & Nodal & plane & & Noda & If plane & $e-2$ \\
\hline No. & $Y$ & $M$ & D & $\mathrm{H}$ & $M$ & $\mathrm{~s}$ & Lat. & Lon. & Dep. & Mag. & Strike & Dip & Rake & Strike & Dip & Rake \\
\hline 1 & 95 & 1 & 28 & 16 & 13 & 24.34 & 34.695 & 135.168 & 9.00 & 2.6 & 270.3 & 28.8 & -36.3 & 32.7 & 73.6 & -114.0 \\
\hline 2 & 95 & 1 & 28 & 19 & 0 & 27.79 & 34.613 & 135.027 & 15.55 & 3.2 & 236.4 & 65.6 & 155.7 & 337.0 & 68.0 & 26.4 \\
\hline 3 & 95 & 1 & 28 & 19 & 55 & 11.24 & 34.613 & 135.023 & 15.92 & 3.4 & 327.9 & 23.1 & 7.1 & 231.4 & 87.2 & 113.0 \\
\hline 4 & 95 & 1 & 29 & 4 & 52 & 36.00 & 34.384 & 134.825 & 8.53 & 3.0 & 289.0 & 72.0 & -2.4 & 19.7 & 87.8 & -161.9 \\
\hline 5 & 95 & 1 & 29 & 6 & 8 & 6.06 & 34.542 & 134.937 & 12.18 & 2.4 & 105.2 & 50.7 & -14.1 & 204.3 & 79.1 & -139.8 \\
\hline 6 & 95 & 1 & 29 & 9 & 41 & 39.05 & 34.673 & 135.141 & 10.13 & 3.0 & 57.0 & 57.6 & -151.2 & 310.6 & 66.0 & -35.9 \\
\hline 7 & 95 & 1 & 29 & 16 & 2 & 45.67 & 34.695 & 135.180 & 8.60 & 3.3 & 245.7 & 75.1 & 173.4 & 337.4 & 83.6 & 15.0 \\
\hline 8 & 95 & 1 & 30 & 4 & 21 & 54.48 & 34.707 & 135.276 & 11.63 & 2.7 & 258.0 & 49.1 & 141.3 & 15.6 & 61.8 & 48.0 \\
\hline 9 & 95 & 1 & 30 & 14 & 43 & 36.09 & 34.543 & 134.893 & 2.16 & 3.2 & 9.5 & 58.7 & 28.6 & 263.7 & 65.9 & 145.3 \\
\hline 10 & 95 & 1 & 30 & 18 & 59 & 14.75 & 34.519 & 135.028 & 10.11 & 2.6 & 181.1 & 32.9 & 135.9 & 310.2 & 67.8 & 65.1 \\
\hline 11 & 95 & 1 & 31 & 1 & 43 & 20.77 & 34.545 & 134.896 & 0.78 & 3.2 & 12.8 & 18.6 & 69.9 & 214.0 & 72.6 & 96.6 \\
\hline 12 & 95 & 1 & 31 & 5 & 36 & 49.31 & 34.699 & 135.189 & 10.15 & 2.4 & 231.7 & 59.5 & 149.2 & 338.5 & 63.8 & 34.4 \\
\hline 13 & 95 & 1 & 31 & 9 & 36 & 6.91 & 34.734 & 135.273 & 5.07 & 2.7 & 154.7 & 46.3 & 46.3 & 28.8 & 58.5 & 125.9 \\
\hline 14 & 95 & 1 & 31 & 11 & 36 & 32.62 & 34.539 & 134.905 & 2.02 & 2.6 & 71.6 & 49.2 & 121.0 & 209.0 & 49.5 & 59.2 \\
\hline 15 & 95 & 1 & 31 & 16 & 18 & 52.76 & 34.944 & 135.376 & 7.11 & 2.4 & 216.5 & 60.5 & -178.6 & 125.8 & 88.8 & -29.5 \\
\hline 16 & 95 & 1 & 31 & 19 & 34 & 9.74 & 34.668 & 135.153 & 9.51 & 2.6 & 77.6 & 42.1 & -106.5 & 279.4 & 50.0 & -75.6 \\
\hline 17 & 95 & 2 & 1 & 1 & 34 & 40.25 & 34.781 & 135.326 & 7.68 & 2.7 & 61.5 & 64.5 & 175.6 & 153.4 & 86.0 & 25.6 \\
\hline 18 & 95 & 2 & 1 & 5 & 41 & 53.98 & 34.561 & 134.989 & 3.79 & 2.5 & 200.8 & 53.3 & 159.3 & 303.5 & 73.5 & 38.5 \\
\hline 19 & 95 & 2 & 1 & 12 & 45 & 49.05 & 34.659 & 135.117 & 8.01 & 2.5 & 304.2 & 57.5 & 28.7 & 197.9 & 66.1 & 144.0 \\
\hline 20 & 95 & 2 & 1 & 18 & 20 & 19.47 & 34.744 & 135.206 & 14.90 & 2.9 & 354.3 & 55.8 & 37.1 & 241.3 & 60.1 & 139.6 \\
\hline 21 & 95 & 2 & 1 & 21 & 48 & 23.92 & 34.515 & 134.898 & 9.62 & 2.5 & 246.9 & 79.3 & -175.7 & 156.1 & 85.8 & -10.7 \\
\hline 22 & 95 & 2 & 1 & 21 & 53 & 55.90 & 34.541 & 134.923 & 6.18 & 2.7 & 279.2 & 40.2 & -172.9 & 183.8 & 85.5 & -50.1 \\
\hline 23 & 95 & 2 & 1 & 22 & 51 & 37.65 & 34.801 & 135.338 & 8.74 & 2.4 & 73.0 & 56.1 & 178.3 & 163.9 & 88.6 & 33.9 \\
\hline 24 & 95 & 2 & 1 & 23 & 30 & 13.56 & 34.435 & 134.872 & 1.66 & 2.6 & 28.6 & 60.6 & 177.5 & 119.8 & 87.8 & 29.5 \\
\hline 25 & 95 & 2 & 2 & 0 & 15 & 47.59 & 34.438 & 134.874 & 1.83 & 2.7 & 49.6 & 36.2 & 178.6 & 140.7 & 89.2 & 53.8 \\
\hline 26 & 95 & 2 & 2 & 1 & 52 & 25.45 & 34.439 & 134.871 & 1.74 & 2.5 & 279.2 & 44.6 & -32.8 & 33.8 & 67.6 & -129.6 \\
\hline 27 & 95 & 2 & 2 & 2 & 33 & 21.56 & 34.560 & 134.946 & 5.40 & 2.5 & 281.4 & 50.3 & -157.3 & 176.5 & 72.7 & -42.0 \\
\hline 28 & 95 & 2 & 2 & 2 & 51 & 3.29 & 34.455 & 134.855 & 10.99 & 2.8 & 231.8 & 58.5 & 166.8 & 328.8 & 78.8 & 32.2 \\
\hline 29 & 95 & 2 & 2 & 6 & 35 & 12.77 & 34.736 & 135.282 & 6.00 & 2.5 & 339.0 & 64.2 & -1.1 & 69.5 & 89.0 & -154.2 \\
\hline 30 & 95 & 2 & 2 & 7 & 39 & 25.65 & 34.700 & 135.187 & 11.28 & 2.6 & 308.6 & 46.5 & 11.7 & 210.4 & 81.5 & 135.9 \\
\hline 31 & 95 & 2 & 2 & 7 & 39 & 54.29 & 34.710 & 135.203 & 11.37 & 2.5 & 283.0 & 31.3 & -154.7 & 171.0 & 77.2 & -61.2 \\
\hline 32 & 95 & 2 & 2 & 9 & 40 & 8.60 & 34.585 & 135.002 & 2.81 & 2.9 & 353.4 & 65.7 & -2.7 & 84.5 & 87.5 & -155.6 \\
\hline 33 & 95 & 2 & 2 & 12 & 1 & 57.31 & 34.854 & 135.219 & 9.34 & 2.5 & 42.6 & 22.0 & 148.6 & 162.1 & 78.7 & 71.0 \\
\hline 34 & 95 & 2 & 2 & 12 & 57 & 22.24 & 34.701 & 135.139 & 5.01 & 2.7 & 136.4 & 40.3 & 22.1 & 29.3 & 75.9 & 128.2 \\
\hline 35 & 95 & 2 & 2 & 14 & 46 & 30.99 & 34.511 & 134.916 & 10.59 & 3.1 & 138.3 & 33.2 & 78.4 & 332.0 & 57.5 & 97.5 \\
\hline 36 & 95 & 2 & 2 & 16 & 4 & 19.91 & 34.589 & 135.040 & 5.64 & 3.6 & 297.8 & 24.0 & 94.3 & 113.2 & 66.1 & 88.1 \\
\hline 37 & 95 & 2 & 2 & 16 & 19 & 27.99 & 34.703 & 135.150 & 17.82 & 4.5 & 208.9 & 45.2 & 119.6 & 350.1 & 51.9 & 63.6 \\
\hline 38 & 95 & 2 & 2 & 20 & 58 & 2.67 & 34.732 & 135.215 & 11.29 & 2.9 & 277.4 & 40.5 & 155.2 & 26.7 & 74.2 & 52.2 \\
\hline 39 & 95 & 2 & 2 & 21 & 32 & 21.03 & 34.707 & 135.189 & 10.21 & 2.5 & 245.4 & 67.5 & 170.1 & 339.3 & 80.8 & 22.8 \\
\hline 40 & 95 & 2 & 2 & 22 & 17 & 29.21 & 34.480 & 134.884 & 4.48 & 2.5 & 144.9 & 68.7 & 15.0 & 49.3 & 76.0 & 158.0 \\
\hline 41 & 95 & 2 & 3 & 1 & 52 & 9.24 & 34.790 & 135.331 & 7.09 & 3.5 & 351.7 & 82.0 & -1.2 & 81.8 & 88.8 & -172.0 \\
\hline 42 & 95 & 2 & 3 & 4 & 36 & 24.05 & 34.706 & 135.188 & 11.98 & 3.9 & 239.2 & 74.5 & -170.3 & 146.6 & 80.7 & -15.7 \\
\hline 43 & 95 & 2 & 3 & 14 & 18 & 31.59 & 34.547 & 134.950 & 9.11 & 3.0 & 279.6 & 72.6 & 174.8 & 11.1 & 85.0 & 17.5 \\
\hline 44 & 95 & 2 & 3 & 20 & 36 & 55.61 & 34.743 & 135.265 & 4.09 & 3.1 & 340.9 & 50.9 & 39.4 & 223.6 & 60.5 & 133.6 \\
\hline 45 & 95 & 2 & 3 & 21 & 22 & 7.11 & 34.590 & 135.001 & 15.71 & 2.9 & 226.7 & 64.8 & 158.2 & 326.4 & 70.3 & 26.9 \\
\hline 46 & 95 & 2 & 3 & 23 & 35 & 29.47 & 34.592 & 135.043 & 6.52 & 3.1 & 94.9 & 44.5 & 126.8 & 228.5 & 55.8 & 59.5 \\
\hline 47 & 95 & 2 & 4 & 0 & 29 & 36.18 & 34.547 & 134.989 & 14.85 & 2.5 & 307.9 & 29.9 & 19.3 & 201.0 & 80.5 & 118.5 \\
\hline 48 & 95 & 2 & 4 & 1 & 32 & 54.85 & 34.606 & 135.032 & 15.60 & 3.1 & 342.6 & 57.9 & 33.1 & 233.5 & 62.5 & 143.2 \\
\hline 49 & 95 & 2 & 4 & 12 & 24 & 21.64 & 34.555 & 135.003 & 11.46 & 2.6 & 73.1 & 40.7 & 137.3 & 198.0 & 63.7 & 57.6 \\
\hline 50 & 95 & 2 & 4 & 16 & 9 & 57.69 & 34.522 & 134.868 & 2.74 & 3.7 & 252.1 & 34.9 & -147.0 & 134.0 & 71.8 & -59.7 \\
\hline 51 & 95 & 2 & 4 & 19 & 38 & 0.26 & 34.523 & 134.890 & 12.40 & 3.7 & 239.3 & 83.0 & 175.9 & 329.8 & 85.9 & 7.0 \\
\hline 52 & 95 & 2 & 5 & 0 & 22 & 7.31 & 34.557 & 134.981 & 8.51 & 2.6 & 206.2 & 71.2 & 161.9 & 302.2 & 72.9 & 19.7 \\
\hline 53 & 95 & 2 & 5 & 4 & 50 & -0.70 & 34.484 & 134.870 & 9.09 & 2.4 & 21.7 & 55.7 & 149.3 & 130.2 & 65.1 & 38.5 \\
\hline 54 & 95 & 2 & 5 & 6 & 44 & 22.69 & 34.701 & 135.181 & 11.53 & 2.7 & 22.5 & 67.2 & -170.9 & 289.0 & 81.6 & -23.1 \\
\hline 55 & 95 & 2 & 5 & 10 & 15 & 0.50 & 34.691 & 135.165 & 10.76 & 2.6 & 301.4 & 76.0 & 8.4 & 209.4 & 81.9 & 165.9 \\
\hline 56 & 95 & 2 & 5 & 10 & 32 & 8.19 & 34.527 & 134.893 & 13.14 & 3.5 & 238.2 & 26.6 & 109.5 & 36.5 & 65.0 & 80.5 \\
\hline 57 & 95 & 2 & 5 & 11 & 12 & 10.62 & 34.678 & 135.141 & 9.31 & 2.4 & 310.4 & 56.1 & 25.4 & 205.5 & 69.2 & 143.4 \\
\hline 58 & 95 & 2 & 5 & 13 & 23 & 39.35 & 34.692 & 135.221 & 10.28 & 2.4 & 270.0 & 42.8 & 42.5 & 90.0 & 47.2 & 123.6 \\
\hline 59 & 95 & 2 & 5 & 15 & 41 & 24.27 & 34.517 & 134.879 & 6.84 & 2.4 & 224.3 & 60.9 & -174.8 & 131.8 & 85.4 & -29.2 \\
\hline 60 & 95 & 2 & 5 & 19 & 46 & 15.73 & 34.729 & 135.208 & 5.46 & 2.6 & 224.9 & 60.0 & -163.4 & 126.4 & 75.6 & -31.0 \\
\hline 61 & 95 & 2 & 6 & 3 & 45 & 34.44 & 34.574 & 134.992 & 6.01 & 3.0 & 212.0 & 38.3 & 55.6 & 73.1 & 59.3 & 114.0 \\
\hline 62 & 95 & 2 & 6 & 13 & 0 & 12.76 & 34.799 & 135.328 & 7.26 & 3.6 & 244.4 & 79.2 & -176.2 & 153.7 & 86.3 & -10.9 \\
\hline 63 & 95 & 2 & 6 & 13 & 2 & 4.39 & & 135 & 7.46 & 2.4 & 164.2 & 51.0 & -17.9 & 265.7 & 76.2 & -139.6 \\
\hline 64 & 95 & 2 & 6 & 16 & 39 & 14.19 & & 134 & 8.52 & 2.6 & 307.8 & 77.0 & 9.9 & 215.6 & 80.4 & 166.8 \\
\hline 65 & 95 & 2 & 6 & 18 & 32 & 38.9 & & 134 & 15.01 & 3.2 & 201.7 & 36.8 & 87.1 & 25.2 & 53.2 & 92.1 \\
\hline 66 & 95 & 2 & 6 & 18 & 45 & 30.26 & & & & 2. & 171.0 & 65.8 & -3.7 & 262.5 & 86.6 & -155.7 \\
\hline 67 & 95 & 2 & 6 & 22 & 11 & & & & & 2. & 105.9 & 21.7 & -107.3 & 304.4 & 69.3 & -83.2 \\
\hline 68 & 95 & 2 & 6 & 22 & 58 & 23.8 & & & & 2. & 71.6 & 58.5 & 170.2 & 166.8 & 81.6 & 31.9 \\
\hline 69 & 95 & 2 & 7 & 8 & 14 & 24.4 & & & & 2. & 318.6 & 49.2 & 7.7 & 223.5 & 84.2 & 138.9 \\
\hline 70 & 95 & 2 & 7 & 8 & 22 & -0.4 & & & & 2. & 44.7 & 78.3 & -168.6 & & 8.9 & -11.9 \\
\hline 71 & 95 & 2 & 7 & 10 & 6 & 36. & & & & 2. & 318.3 & 61.3 & 7.1 & 224.8 & 3.8 & 151.1 \\
\hline 72 & 95 & 2 & 7 & 21 & 34 & 19.37 & & 134.868 & 5.49 & 2.6 & 321.8 & 65.5 & -8.5 & 55.3 & 82.3 & -155.3 \\
\hline 73 & 95 & 2 & 7 & 23 & 45 & 47.60 & 34.531 & 134.994 & 14.71 & 2.7 & 128.7 & 39.2 & 76.3 & 326.2 & 52.1 & 101.0 \\
\hline 74 & 95 & 2 & 8 & 0 & 15 & 20.87 & 34.523 & 134.904 & 9.78 & 3.1 & 235.2 & 72.0 & 177.5 & 326.0 & 87.6 & 18.0 \\
\hline 75 & 95 & 2 & 8 & 4 & 38 & 55.61 & 34.520 & 134.913 & 11.16 & 2.5 & 159.5 & 48.3 & 27.9 & 50.1 & 69.6 & 134.8 \\
\hline 76 & 95 & 2 & 8 & 9 & 19 & 13.33 & 34.529 & 134.999 & 14.93 & 2.6 & 158.3 & 37.6 & 92.8 & 334.7 & 52.5 & 87.8 \\
\hline 77 & 95 & 2 & 8 & 13 & 46 & 26.42 & 34.882 & 135.359 & 1.86 & 2.4 & 196.9 & 76.6 & -167.6 & 103.9 & 77.9 & -13.7 \\
\hline 78 & 95 & 2 & 8 & 15 & 40 & 32.43 & 34.631 & 135.108 & 8.47 & 3.1 & 164.6 & 55.1 & 23.9 & 60.4 & 70.6 & 142.7 \\
\hline 79 & 95 & 2 & 9 & 3 & 52 & 58.02 & 34.796 & 135.343 & 7.53 & 2.5 & 284.8 & 50.8 & -31.6 & 36.1 & 66.1 & -136.2 \\
\hline 80 & 95 & 2 & 9 & 7 & 17 & 28.48 & 34.736 & 135.212 & 9.78 & 2.4 & 253.6 & 23.6 & 130.5 & 30.6 & 72.3 & 74.2 \\
\hline
\end{tabular}


Table 1. (continued).

\begin{tabular}{|c|c|c|c|c|c|c|c|c|c|c|c|c|c|c|c|c|}
\hline & & & & & & & & & & & Nodal & plane & & Noda & I plane & -2 \\
\hline No. & $Y$ & M & D & $\mathrm{H}$ & $M$ & $\mathrm{~S}$ & Lat. & Lon. & Dep. & Mag. & Strike & Dip & Rake & Strike & Dip & Rake \\
\hline 81 & 95 & 2 & 9 & 13 & 19 & -0.39 & 34.531 & 134.919 & 14.49 & 2.7 & 224.9 & 78.5 & 180.0 & 314.9 & 90.0 & 11.5 \\
\hline 82 & 95 & 2 & 9 & 14 & 40 & 25.89 & 34.525 & 134.887 & 9.18 & 2.8 & 229.9 & 65.8 & 170.7 & 323.8 & 81.6 & 24.5 \\
\hline 83 & 95 & 2 & 9 & 16 & 2 & 15.48 & 34.451 & 134.817 & 1.00 & 2.8 & 33.1 & 74.2 & 170.9 & 125.6 & 81.3 & 16.0 \\
\hline 84 & 95 & 2 & 10 & 3 & 18 & 36.73 & 34.779 & 135.363 & 2.63 & 2.9 & 62.5 & 67.9 & -173.2 & 329.9 & 83.7 & -22.3 \\
\hline 85 & 95 & 2 & 10 & 6 & 39 & 3.10 & 34.529 & 134.996 & 14.97 & 2.9 & 135.7 & 33.9 & 69.0 & 340.6 & 58.7 & 103.5 \\
\hline 86 & 95 & 2 & 10 & 15 & 19 & 48.79 & 34.559 & 135.039 & 5.77 & 3.0 & 30.9 & 74.5 & 170.3 & 123.5 & 80.7 & 15.7 \\
\hline 87 & 95 & 2 & 11 & 2 & 25 & 40.32 & 34.572 & 134.982 & 9.41 & 2.9 & 274.6 & 37.2 & 145.8 & 33.0 & 70.2 & 57.9 \\
\hline 88 & 95 & 2 & 11 & 5 & 20 & 47.03 & 34.769 & 135.262 & 6.58 & 2.6 & 224.8 & 54.6 & 168.9 & 321.2 & 81.0 & 35.9 \\
\hline 89 & 95 & 2 & 11 & 14 & 29 & 7.20 & 34.549 & 135.018 & 3.30 & 2.4 & 332.8 & 47.5 & 61.6 & 191.4 & 49.6 & 117.4 \\
\hline 90 & 95 & 2 & 12 & 6 & 11 & 22.49 & 34.854 & 135.220 & 8.62 & 2.5 & 332.9 & 28.5 & 86.4 & 157.0 & 61.6 & 92.0 \\
\hline 91 & 95 & 2 & 12 & 8 & 24 & 8.25 & 34.852 & 135.220 & 9.26 & 2.4 & 295.3 & 22.0 & 43.8 & 163.7 & 75.0 & 106.2 \\
\hline 92 & 95 & 2 & 12 & 8 & 31 & 16.43 & 34.553 & 134.947 & 8.06 & 2.4 & 168.6 & 45.7 & 7.3 & 73.5 & 84.8 & 135.5 \\
\hline 93 & 95 & 2 & 12 & 16 & 25 & 48.71 & 34.546 & 134.902 & 11.10 & 2.7 & 255.0 & 82.2 & 177.9 & 345.3 & 87.9 & 7.8 \\
\hline 94 & 95 & 2 & 12 & 17 & 48 & -0.15 & 34.717 & 135.211 & 10.13 & 2.5 & 242.8 & 58.8 & -159.2 & 141.7 & 72.3 & -32.9 \\
\hline 95 & 95 & 2 & 12 & 18 & 4 & 23.98 & 34.522 & 134.892 & 14.06 & 3.2 & 164.4 & 60.9 & -23.8 & 266.4 & 69.4 & -148.7 \\
\hline 96 & 95 & 2 & 13 & 5 & 1 & 8.81 & 34.825 & 135.320 & 6.08 & 2.6 & 200.2 & 65.4 & 163.1 & 297.4 & 74.7 & 25.6 \\
\hline 97 & 95 & 2 & 13 & 10 & 8 & 6.62 & 34.546 & 135.018 & 2.63 & 2.5 & 184.8 & 14.3 & 113.9 & 340.2 & 76.9 & 84.1 \\
\hline 98 & 95 & 2 & 13 & 20 & 23 & 55.45 & 34.965 & 135.324 & 8.43 & 3.5 & 214.6 & 68.9 & 164.8 & 310.2 & 75.8 & 21.8 \\
\hline 99 & 95 & 2 & 13 & 20 & 45 & 49.74 & 34.542 & 134.889 & 8.98 & 2.9 & 238.1 & 67.5 & -174.9 & 146.2 & 85.3 & -22.6 \\
\hline 100 & 95 & 2 & 14 & 2 & 50 & 49.06 & 34.531 & 134.927 & 14.72 & 3.1 & 314.6 & 78.3 & 11.3 & 222.3 & 78.9 & 168.0 \\
\hline 101 & 95 & 2 & 14 & 10 & 32 & 45.41 & 34.735 & 135.211 & 11.19 & 2.7 & 289.6 & 48.0 & -174.4 & 195.9 & 85.8 & -42.1 \\
\hline 102 & 95 & 2 & 15 & 0 & 3 & 22.88 & 34.667 & 135.138 & 9.41 & 2.7 & 330.2 & 52.0 & 36.9 & 215.3 & 61.7 & 135.7 \\
\hline 103 & 95 & 2 & 15 & 1 & 3 & 25.18 & 34.698 & 135.178 & 10.42 & 2.5 & 284.6 & 21.2 & -14.1 & 27.8 & 84.9 & -110.6 \\
\hline 104 & 95 & 2 & 15 & 1 & 39 & 52.56 & 34.967 & 135.324 & 8.92 & 2.6 & 307.5 & 68.1 & -14.1 & 42.9 & 76.9 & -157.5 \\
\hline 105 & 95 & 2 & 15 & 14 & 44 & 27.75 & 34.508 & 134.893 & 9.43 & 3.3 & 120.9 & 41.1 & 16.5 & 18.3 & 79.3 & 129.9 \\
\hline 106 & 95 & 2 & 15 & 21 & 5 & 44.77 & 34.447 & 134.832 & 3.95 & 2.7 & 239.8 & 48.6 & 143.1 & 356.2 & 63.3 & 47.8 \\
\hline 107 & 95 & 2 & 15 & 22 & 45 & 39.35 & 34.548 & 135.017 & 2.83 & 2.7 & 223.4 & 16.9 & 128.3 & 3.9 & 76.8 & 79.3 \\
\hline 108 & 95 & 2 & 16 & 12 & 57 & 19.52 & 34.694 & 135.183 & 8.43 & 3.1 & 313.7 & 66.8 & -11.7 & 48.4 & 79.2 & -156.4 \\
\hline 109 & 95 & 2 & 16 & 22 & 25 & 48.56 & 34.665 & 135.136 & 10.10 & 2.9 & 296.2 & 62.8 & 21.1 & 196.2 & 71.3 & 151.2 \\
\hline 110 & 95 & 2 & 17 & 0 & 10 & 26.69 & 34.454 & 134.850 & 2.51 & 2.4 & 153.6 & 42.5 & 114.5 & 301.9 & 52.1 & 69.2 \\
\hline 111 & 95 & 2 & 17 & 3 & 9 & 33.19 & 34.436 & 134.815 & 2.00 & 3.1 & 338.9 & 41.7 & 34.2 & 222.0 & 68.0 & 126.4 \\
\hline 112 & 95 & 2 & 17 & 8 & 52 & 0.13 & 34.739 & 135.216 & 7.80 & 2.4 & 141.4 & 41.5 & -5.7 & 235.7 & 86.2 & -131.4 \\
\hline 113 & 95 & 2 & 17 & 20 & 13 & 1.62 & 34.559 & 134.979 & 2.15 & 2.5 & 15.1 & 37.7 & 64.8 & 225.8 & 56.4 & 108.2 \\
\hline 114 & 95 & 2 & 18 & 1 & 41 & 24.56 & 34.576 & 135.046 & 6.34 & 2.4 & 323.8 & 58.5 & 150.3 & 70.4 & 65.0 & 35.3 \\
\hline 115 & 95 & 2 & 18 & 2 & 0 & 51.97 & 34.833 & 135.364 & 8.88 & 2.9 & 323.5 & 72.1 & -3.1 & 54.4 & 87.0 & -162.0 \\
\hline 116 & 95 & 2 & 18 & 5 & 49 & 7.49 & 34.534 & 134.876 & 1.66 & 3.1 & 136.2 & 44.0 & -88.4 & 313.9 & 46.0 & -91.6 \\
\hline 117 & 95 & 2 & 18 & 12 & 12 & 45.71 & 34.692 & 135.185 & 7.70 & 2.6 & 345.2 & 42.3 & 28.7 & 233.1 & 71.1 & 128.6 \\
\hline 118 & 95 & 2 & 18 & 19 & 29 & 28.96 & 34.607 & 135.026 & 15.55 & 2.8 & 340.9 & 61.8 & 24.8 & 238.6 & 68.3 & 149.4 \\
\hline 119 & 95 & 2 & 18 & 21 & 1 & 4.90 & 34.793 & 135.330 & 8.18 & 2.4 & 173.9 & 61.5 & 21.1 & 73.5 & 71.6 & 149.8 \\
\hline 120 & 95 & 2 & 18 & 21 & 37 & 33.87 & 34.431 & 134.804 & 12.88 & 3.9 & 145.2 & 72.0 & -2.6 & 236.0 & 87.6 & -162.0 \\
\hline 121 & 95 & 2 & 18 & 23 & 16 & 14.66 & 34.734 & 135.272 & 5.15 & 2.5 & 350.6 & 34.0 & 71.2 & 192.9 & 58.0 & 102.2 \\
\hline 122 & 95 & 2 & 19 & 1 & 19 & 6.39 & 34.439 & 134.814 & 11.55 & 3.0 & 141.4 & 77.2 & -10.1 & 233.6 & 80.2 & -167.0 \\
\hline 123 & 95 & 2 & 19 & 5 & 51 & 28.19 & 34.437 & 134.806 & 11.83 & 3.6 & 221.6 & 77.7 & -169.3 & 129.3 & 79.5 & -12.5 \\
\hline 124 & 95 & 2 & 19 & 7 & 2 & 37.41 & 34.782 & 135.320 & 9.22 & 2.8 & 69.7 & 50.4 & 166.7 & 168.3 & 79.8 & 40.4 \\
\hline 125 & 95 & 2 & 19 & 20 & 15 & 9.00 & 34.541 & 134.893 & 9.12 & 2.5 & 217.4 & 17.7 & 128.7 & 357.3 & 76.3 & 78.7 \\
\hline 126 & 95 & 2 & 19 & 20 & 27 & 15.71 & 34.539 & 134.897 & 9.06 & 3.4 & 151.0 & 48.9 & 5.2 & 57.6 & 86.1 & 138.7 \\
\hline 127 & 95 & 2 & 20 & 5 & 43 & 39.95 & 34.692 & 135.160 & 15.89 & 2.9 & 214.9 & 15.9 & 161.1 & 323.1 & 84.9 & 74.9 \\
\hline 128 & 95 & 2 & 20 & 14 & 41 & 2.02 & 34.581 & 135.008 & 8.30 & 2.7 & 147.2 & 55.7 & 6.9 & 53.2 & 84.3 & 145.5 \\
\hline 129 & 95 & 2 & 20 & 19 & 8 & 16.16 & 34.741 & 135.275 & 3.99 & 2.6 & 29.4 & 44.6 & 119.9 & 170.4 & 52.5 & 63.8 \\
\hline 130 & 95 & 2 & 20 & 19 & 36 & 42.26 & 34.591 & 135.054 & 5.94 & 2.4 & 39.2 & 47.1 & 29.1 & 288.5 & 69.2 & 133.2 \\
\hline 131 & 95 & 2 & 21 & 3 & 53 & 5.03 & 34.781 & 135.318 & 9.01 & 2.6 & 148.5 & 60.5 & 1.1 & 58.0 & 89.0 & 150.5 \\
\hline 132 & 95 & 2 & 21 & 6 & 36 & 54.69 & 34.606 & 135.040 & 14.91 & 3.2 & 334.0 & 63.9 & 14.4 & 237.6 & 77.1 & 153.2 \\
\hline 133 & 95 & 2 & 21 & 7 & 15 & 7.60 & 34.685 & 135.184 & 8.02 & 2.4 & 343.1 & 38.2 & 17.4 & 239.2 & 79.3 & 126.9 \\
\hline 134 & 95 & 2 & 21 & 12 & 34 & 56.80 & 34.734 & 135.265 & 3.92 & 2.3 & 164.5 & 35.8 & 71.1 & 7.4 & 56.4 & 103.2 \\
\hline 135 & 95 & 2 & 22 & 5 & 56 & 38.59 & 34.461 & 134.845 & 8.53 & 3.1 & 245.3 & 60.8 & -175.6 & 153.2 & 86.1 & -29.3 \\
\hline 136 & 95 & 2 & 22 & 22 & 28 & 12.02 & 34.797 & 135.339 & 8.79 & 2.8 & 153.2 & 90.0 & 0.0 & 63.2 & 90.0 & 180.0 \\
\hline 137 & 95 & 2 & 24 & 8 & 3 & 48.09 & 34.718 & 135.215 & 11.66 & 3.1 & 43.8 & 42.9 & 88.3 & 226.1 & 47.2 & 91.6 \\
\hline 138 & 95 & 2 & 24 & 12 & 15 & 53.93 & 34.796 & 135.337 & 8.84 & 2.7 & 67.6 & 79.4 & -175.6 & 336.7 & 85.7 & -10.7 \\
\hline 139 & 95 & 2 & 24 & 16 & 41 & 53.05 & 34.664 & 135.149 & 7.96 & 2.6 & 320.0 & 53.9 & -8.0 & 54.7 & 83.5 & -143.6 \\
\hline 140 & 95 & 2 & 25 & 4 & 44 & 58.41 & 34.743 & 135.267 & 6.32 & 2.7 & 11.3 & 44.5 & 105.7 & 169.8 & 47.5 & 75.1 \\
\hline 141 & 95 & 2 & 25 & 12 & 59 & 20.69 & 34.540 & 134.993 & 14.93 & 3.3 & 142.1 & 46.1 & 73.9 & 344.7 & 46.2 & 106.1 \\
\hline 142 & 95 & 2 & 25 & 14 & 24 & 53.36 & 34.578 & 134.961 & 0.82 & 9.9 & 26.2 & 38.1 & 92.8 & 202.6 & 52.0 & 87.8 \\
\hline 143 & 95 & 2 & 25 & 16 & 53 & 55.64 & 34.528 & 134.925 & 14.37 & 3.0 & 234.0 & 68.5 & -165.3 & 138.5 & 76.4 & -22.2 \\
\hline 144 & 95 & 2 & 27 & 5 & 31 & 33.66 & 34.491 & 134.914 & 3.27 & 2.5 & 314.1 & 39.6 & 36.8 & 194.2 & 67.6 & 123.5 \\
\hline 145 & 95 & 2 & 27 & 22 & 17 & 38.43 & & & 2.5 & 2.8 & 233.6 & 72.1 & 176.9 & 324.6 & 87.1 & 18.0 \\
\hline 146 & 95 & 2 & 27 & 22 & 45 & 35.7 & & & & 2.5 & 5.6 & 71.8 & 0.7 & 245.4 & 89.4 & 161.8 \\
\hline 147 & 95 & 2 & 28 & 11 & 16 & 24.4 & & & & 2. & 143.1 & 62.4 & 20.6 & 43.3 & 71.8 & 150.9 \\
\hline 148 & 95 & 3 & 1 & 5 & 51 & 28.8 & & & & 2. & 180.0 & 37.9 & 90.0 & 360.0 & 52.1 & 90.0 \\
\hline 149 & 95 & 3 & 2 & 6 & 24 & 6.23 & & & & 3. & 87.8 & 46.3 & 131.6 & 215.7 & 57.2 & 55.2 \\
\hline 150 & 95 & 3 & 2 & 6 & 39 & 7.70 & & & & & 165.8 & 50.4 & 139.9 & 284.0 & 60.2 & 47.2 \\
\hline 151 & 95 & 3 & 2 & 16 & 53 & 18.07 & & 134.8 & 10.2 & 2. & 120.6 & 49.4 & 12.8 & 22.2 & 80.3 & 138.7 \\
\hline 152 & 95 & 3 & 3 & 5 & 12 & 26.34 & 34.534 & 134.999 & 14.7 & & 192.8 & 64.5 & 152.4 & 295.5 & 65.3 & 28.2 \\
\hline 153 & 95 & 3 & 3 & 11 & 56 & 12.15 & 34.565 & 134.967 & & 2.7 & 237.5 & 52.0 & 134.3 & 359.8 & 55.6 & 48.2 \\
\hline 154 & 95 & 3 & 4 & 20 & 39 & 42.13 & 34.497 & 134.974 & & 2.5 & 199.6 & 21.6 & 85.3 & 24.6 & 68.5 & 91.8 \\
\hline 155 & 95 & 3 & 5 & 0 & 36 & 24.40 & 34.603 & 135 & & 2.8 & 204.6 & 40.5 & 80.2 & 37.3 & 50.2 & 98.2 \\
\hline 156 & 95 & 3 & 5 & 6 & 51 & 53.13 & 34.766 & 135.294 & 6.20 & 2.7 & 359.2 & 33.1 & 44.4 & 229.9 & 67.6 & 115.0 \\
\hline 157 & 95 & 3 & 5 & 10 & 4 & 28.73 & 34.745 & 135.245 & 10.99 & 3.0 & 157.7 & 41.7 & 77.0 & 354.9 & 49.6 & 101.4 \\
\hline 158 & 95 & 3 & 5 & 15 & 14 & 47.16 & 34.766 & 135.294 & 6.01 & 2.8 & 181.3 & 34.2 & 46.1 & 50.6 & 66.1 & 115.3 \\
\hline 159 & 95 & 3 & 5 & 16 & 29 & 33.28 & 34.543 & 134.934 & 6.53 & 2.4 & 162.0 & 53.4 & -174.9 & 68.9 & 85.9 & -36.7 \\
\hline 160 & 95 & 3 & 5 & 16 & 33 & 37.46 & 34.528 & 135.070 & 14.34 & 2.6 & 319.0 & 61.2 & 18.6 & 219.8 & 73.7 & 149.8 \\
\hline
\end{tabular}


Table 1. (continued).

\begin{tabular}{|c|c|c|c|c|c|c|c|c|c|c|c|c|c|c|c|c|}
\hline & & & & & & & & & & & Nodal & plane & -1 & Noda & al plane & $e-2$ \\
\hline No. & $Y$ & M & D & $\mathrm{H}$ & $M$ & S & Lat. & Lon. & Dep. & Mag. & Strike & Dip & Rake & Strike & Dip & Rake \\
\hline 161 & 95 & 3 & 6 & 19 & 7 & 53.10 & 34.481 & 134.873 & 9.63 & 3.2 & 126.7 & 90.0 & 0.0 & 36.7 & 90.0 & 180.0 \\
\hline 162 & 95 & 3 & 7 & 16 & 20 & 26.25 & 34.565 & 134.993 & 6.14 & 2.5 & 64.1 & 33.5 & 129.4 & 199.6 & 64.7 & 67.2 \\
\hline 163 & 95 & 3 & 8 & 3 & 18 & 46.31 & 34.493 & 134.982 & 3.92 & 2.8 & 206.0 & 22.2 & 44.4 & 73.9 & 74.7 & 106.3 \\
\hline 164 & 95 & 3 & 8 & 3 & 29 & 29.49 & 34.494 & 134.982 & 3.76 & 2.6 & 190.1 & 6.5 & 28.4 & 71.9 & 86.9 & 95.7 \\
\hline 165 & 95 & 3 & 8 & 20 & 5 & 0.55 & 34.450 & 134.784 & 1.54 & 2.7 & 246.2 & 55.2 & 149.8 & 354.6 & 65.6 & 38.8 \\
\hline 166 & 95 & 3 & 8 & 23 & 11 & 7.02 & 34.688 & 135.192 & 9.28 & 2.6 & 39.4 & 43.1 & -133.5 & 271.8 & 60.3 & -57.2 \\
\hline 167 & 95 & 3 & 9 & 5 & 18 & 36.55 & 34.881 & 135.347 & 1.07 & 2.4 & 199.5 & 66.8 & -172.1 & 106.3 & 82.7 & -23.4 \\
\hline 168 & 95 & 3 & 9 & 8 & 35 & 2.33 & 34.530 & 134.925 & 14.04 & 2.4 & 163.7 & 25.1 & 50.4 & 26.1 & 70.9 & 106.6 \\
\hline 169 & 95 & 3 & 9 & 20 & 32 & 39.29 & 34.648 & 135.133 & 8.18 & 3.0 & 339.3 & 50.5 & -13.5 & 78.0 & 79.6 & -139.7 \\
\hline 170 & 95 & 3 & 10 & 0 & 33 & 56.65 & 34.621 & 135.097 & 9.51 & 2.6 & 318.9 & 42.7 & 13.4 & 219.0 & 81.0 & 131.9 \\
\hline 171 & 95 & 3 & 11 & 9 & 41 & 36.34 & 34.513 & 135.007 & 14.19 & 3.1 & 301.2 & 48.9 & 132.6 & 66.7 & 56.3 & 52.2 \\
\hline 172 & 95 & 3 & 12 & 0 & 32 & 49.06 & 34.427 & 134.769 & 4.36 & 2.4 & 333.9 & 33.4 & 0.4 & 243.6 & 89.8 & 123.4 \\
\hline 173 & 95 & 3 & 12 & 20 & 17 & 25.41 & 34.880 & 135.352 & 1.61 & 2.9 & 184.6 & 56.5 & 174.7 & 277.5 & 85.6 & 33.7 \\
\hline 174 & 95 & 3 & 13 & 7 & 30 & 38.11 & 34.537 & 134.902 & 4.39 & 2.4 & 344.7 & 44.6 & 38.5 & 225.2 & 64.1 & 127.7 \\
\hline 175 & 95 & 3 & 14 & 1 & 14 & 1.19 & 34.936 & 135.340 & 11.55 & 2.7 & 18.2 & 51.5 & 140.1 & 135.7 & 59.8 & 46.1 \\
\hline 176 & 95 & 3 & 14 & 20 & 59 & 42.55 & 34.598 & 135.050 & 5.64 & 2.4 & 227.6 & 23.9 & 92.8 & 44.5 & 66.2 & 88.7 \\
\hline 177 & 95 & 3 & 15 & 10 & 39 & 8.44 & 34.542 & 134.867 & 0.84 & 2.6 & 3.3 & 46.8 & 25.0 & 255.6 & 72.0 & 133.9 \\
\hline 178 & 95 & 3 & 16 & 9 & 30 & 21.14 & 34.549 & 134.980 & 8.28 & 2.5 & 198.1 & 61.4 & 172.4 & 291.7 & 83.3 & 28.8 \\
\hline 179 & 95 & 3 & 16 & 13 & 54 & 51.35 & 34.426 & 134.774 & 4.82 & 3.2 & 200.0 & 34.6 & 60.5 & 54.5 & 60.4 & 108.8 \\
\hline 180 & 95 & 3 & 17 & 10 & 40 & 2.24 & 34.509 & 134.993 & 15.58 & 2.5 & 150.1 & 39.2 & 103.7 & 312.6 & 52.2 & 79.0 \\
\hline 181 & 95 & 3 & 18 & 14 & 54 & 12.20 & 34.643 & 135.125 & 12.22 & 2.6 & 65.8 & 45.0 & 81.9 & 257.2 & 45.6 & 98.0 \\
\hline 182 & 95 & 3 & 18 & 20 & 30 & 6.06 & 34.880 & 135.351 & 2.22 & 2.4 & 302.8 & 57.5 & 10.3 & 207.2 & 81.3 & 147.1 \\
\hline 183 & 95 & 3 & 18 & 23 & 43 & 31.93 & 34.875 & 135.301 & 3.78 & 2.8 & 152.5 & 44.1 & 113.7 & 301.0 & 50.4 & 68.7 \\
\hline 184 & 95 & 3 & 20 & 20 & 3 & 7.98 & 34.739 & 135.262 & 1.87 & 2.5 & 218.4 & 44.7 & 106.5 & 15.8 & 47.5 & 74.3 \\
\hline 185 & 95 & 3 & 22 & 18 & 1 & 6.51 & 34.539 & 134.895 & 10.16 & 2.5 & 246.6 & 70.2 & 163.1 & 342.5 & 74.1 & 20.7 \\
\hline 186 & 95 & 3 & 23 & 0 & 49 & 43.95 & 34.556 & 134.966 & 6.60 & 2.8 & 214.6 & 45.3 & 25.8 & 105.8 & 71.9 & 132.3 \\
\hline 187 & 95 & 3 & 23 & 7 & 7 & 56.98 & 34.512 & 134.912 & 10.45 & 2.5 & 126.8 & 41.3 & 44.6 & 0.2 & 62.4 & 122.0 \\
\hline 188 & 95 & 3 & 25 & 0 & 50 & 17.71 & 34.462 & 134.837 & 10.13 & 2.8 & 64.8 & 61.6 & -147.3 & 317.8 & 61.6 & -32.7 \\
\hline 189 & 95 & 3 & 25 & 11 & 31 & 15.09 & 34.887 & 135.396 & 11.60 & 2.4 & 293.9 & 29.0 & -8.6 & 31.4 & 85.8 & -118.8 \\
\hline 190 & 95 & 3 & 27 & 0 & 30 & 19.69 & 34.783 & 135.340 & 12.34 & 2.5 & 67.4 & 71.8 & -178.8 & 337.0 & 88.8 & -18.2 \\
\hline 191 & 95 & 3 & 28 & 7 & 23 & 8.29 & 34.869 & 135.397 & 13.11 & 2.5 & 37.2 & 39.1 & 142.5 & 158.0 & 67.4 & 57.2 \\
\hline 192 & 95 & 3 & 28 & 11 & 56 & 49.18 & 34.876 & 135.361 & -0.53 & 2.9 & 17.8 & 77.1 & 167.1 & 110.7 & 77.4 & 13.3 \\
\hline 193 & 95 & 3 & 29 & 1 & 14 & 0.43 & 34.765 & 135.343 & 10.29 & 2.4 & 241.7 & 75.6 & 172.4 & 333.6 & 82.6 & 14.5 \\
\hline 194 & 95 & 3 & 29 & 14 & 57 & 27.99 & 34.516 & 134.917 & 5.26 & 2.3 & 319.1 & 32.4 & 28.8 & 204.1 & 75.0 & 119.1 \\
\hline 195 & 95 & 3 & 30 & 14 & 42 & 1.63 & 34.788 & 135.319 & 8.34 & 2.5 & 50.0 & 40.8 & 139.9 & 172.6 & 65.1 & 56.5 \\
\hline 196 & 95 & 3 & 31 & 5 & 10 & 33.44 & 34.756 & 135.301 & 4.20 & 2.8 & 17.7 & 41.4 & 66.8 & 227.4 & 52.6 & 109.1 \\
\hline 197 & 95 & 4 & 1 & 3 & 53 & 33.71 & 34.482 & 134.943 & 7.03 & 2.6 & 158.7 & 41.3 & 44.6 & 32.2 & 62.3 & 122.1 \\
\hline 198 & 95 & 4 & 2 & 13 & 28 & 1.69 & 34.553 & 134.983 & 13.26 & 2.5 & 259.7 & 32.3 & 119.1 & 46.3 & 62.2 & 72.9 \\
\hline 199 & 95 & 4 & 2 & 14 & 33 & 26.60 & 34.650 & 135.157 & 8.45 & 3.0 & 302.4 & 32.4 & 20.9 & 194.6 & 79.0 & 120.6 \\
\hline 200 & 95 & 4 & 3 & 1 & 45 & 0.52 & 34.881 & 135.333 & 1.75 & 2.5 & 121.7 & 49.6 & 13.6 & 22.8 & 79.7 & 138.8 \\
\hline 201 & 95 & 4 & 3 & 11 & 14 & 12.25 & 34.559 & 134.987 & 8.38 & 2.3 & 263.6 & 81.0 & 7.1 & 172.5 & 83.0 & 170.9 \\
\hline 202 & 95 & 4 & 3 & 22 & 31 & 45.35 & 34.529 & 134.926 & 14.76 & 2.7 & 328.4 & 57.5 & 18.4 & 228.2 & 74.5 & 146.1 \\
\hline 203 & 95 & 4 & 4 & 1 & 36 & 22.30 & 34.604 & 135.066 & 9.42 & 2.8 & 72.7 & 52.8 & 145.9 & 184.9 & 63.5 & 42.5 \\
\hline 204 & 95 & 4 & 4 & 1 & 39 & 5.54 & 34.769 & 135.294 & 5.11 & 2.3 & 168.9 & 38.1 & 99.3 & 337.1 & 52.5 & 82.8 \\
\hline 205 & 95 & 4 & 4 & 2 & 50 & 2.84 & 34.729 & 135.273 & 1.30 & 2.6 & 185.9 & 40.7 & 106.0 & 345.2 & 51.2 & 76.6 \\
\hline 206 & 95 & 4 & 5 & 2 & 12 & 24.16 & 34.784 & 135.327 & 4.71 & 2.8 & 159.6 & 40.5 & -53.0 & 294.9 & 58.8 & -117.1 \\
\hline 207 & 95 & 4 & 6 & 10 & 50 & 48.62 & 34.793 & 135.326 & 6.41 & 2.7 & 82.4 & 69.5 & -158.2 & 344.5 & 69.7 & -21.9 \\
\hline 208 & 95 & 4 & 6 & 10 & 55 & 4.87 & 34.790 & 135.328 & 6.45 & 2.6 & 271.2 & 73.8 & -171.6 & 178.8 & 82.0 & -16.4 \\
\hline 209 & 95 & 4 & 6 & 11 & 0 & 8.05 & 34.789 & 135.329 & 5.55 & 2.6 & 250.0 & 37.6 & -165.0 & 148.0 & 80.9 & -53.3 \\
\hline 210 & 95 & 4 & 6 & 20 & 22 & 6.03 & 34.794 & 135.328 & 6.62 & 2.3 & 260.5 & 37.2 & -172.8 & 164.8 & 85.7 & -53.0 \\
\hline 211 & 95 & 4 & 7 & 1 & 9 & 31.36 & 34.523 & 134.920 & 6.00 & 2.9 & 301.5 & 36.4 & -5.8 & 36.1 & 86.6 & -126.2 \\
\hline 212 & 95 & 4 & 7 & 4 & 34 & 28.11 & 34.660 & 135.165 & 8.85 & 2.8 & 231.0 & 40.0 & 103.5 & 33.6 & 51.3 & 78.9 \\
\hline 213 & 95 & 4 & 7 & 10 & 8 & 38.87 & 34.791 & 135.325 & 6.23 & 2.5 & 259.1 & 43.8 & -148.4 & 145.2 & 68.8 & -50.7 \\
\hline 214 & 95 & 4 & 10 & 21 & 17 & 34.23 & 34.433 & 134.820 & 14.14 & 3.3 & 171.9 & 45.3 & 119.5 & 313.1 & 51.8 & 63.5 \\
\hline 215 & 95 & 4 & 11 & 7 & 38 & 2.19 & 34.554 & 134.983 & 8.79 & 3.0 & 280.2 & 57.5 & 5.5 & 187.2 & 85.4 & 147.4 \\
\hline 216 & 95 & 4 & 12 & 13 & 40 & 0.57 & 34.561 & 134.977 & 14.38 & 2.5 & 130.4 & 60.6 & 3.3 & 38.8 & 87.1 & 150.6 \\
\hline 217 & 95 & 4 & 12 & 23 & 2 & 37.74 & 34.782 & 135.321 & 7.79 & 2.8 & 157.8 & 79.4 & 4.4 & 67.0 & 85.7 & 169.4 \\
\hline 218 & 95 & 4 & 13 & 5 & 49 & 33.59 & 34.607 & 135.032 & 15.53 & 2.4 & 240.7 & 36.0 & 148.2 & 357.3 & 71.9 & 58.3 \\
\hline 219 & 95 & 4 & 13 & 22 & 29 & 32.01 & 34.880 & 135.355 & 1.59 & 2.6 & 305.2 & 54.2 & 22.4 & 201.6 & 72.0 & 142.1 \\
\hline 220 & 95 & 4 & 14 & 16 & 33 & 15.27 & 34.770 & 135.284 & 9.53 & 2.5 & 230.2 & 16.3 & -175.0 & 135.4 & 88.6 & -73.7 \\
\hline 221 & 95 & 4 & 15 & 7 & 29 & 42.78 & 34.531 & 134.928 & 3.19 & 2.3 & 171.5 & 21.8 & -160.1 & 63.0 & 82.7 & -69.3 \\
\hline 222 & 95 & 4 & 15 & 19 & 26 & 28.61 & 34.782 & 135.326 & 7.50 & 2.5 & 310.8 & 29.6 & -13.6 & 52.6 & 83.3 & -118.9 \\
\hline 223 & 95 & 4 & 15 & 19 & 55 & 25.41 & 34.784 & 135.325 & 6.96 & 2.4 & 310.2 & 52.2 & -35.9 & 64.1 & 62.4 & -136.3 \\
\hline 224 & 95 & 4 & 16 & 3 & 55 & 36.02 & 34.787 & 135.326 & 7.01 & 2.4 & 164.5 & 66.8 & 18.8 & 66.8 & 72.8 & 155.6 \\
\hline 225 & 95 & 4 & 16 & 5 & 34 & 51.02 & 34.641 & 135.272 & 13.65 & 2.3 & 322.2 & 43.4 & 66.9 & 172.7 & 50.8 & 110.4 \\
\hline 226 & 95 & 4 & 19 & 21 & 41 & 19.32 & 34.763 & 135.287 & 3.56 & 2.5 & 22.7 & 26.3 & 70.2 & 224.6 & 65.4 & 99.5 \\
\hline 227 & 95 & 4 & 20 & 17 & 42 & 24.38 & 34.450 & 134.779 & 1.69 & 2.3 & 295.0 & 42.3 & -99.4 & 127.6 & 48.4 & -81.5 \\
\hline 228 & 95 & 4 & 23 & 8 & 43 & 17.09 & 34.738 & 135.262 & 10.64 & 2.5 & 211.6 & 42.0 & 121.5 & 352.1 & 55.2 & 64.8 \\
\hline 229 & 95 & 4 & 23 & 8 & 59 & 57.71 & 34.522 & 134.878 & 2.79 & 2.7 & 133.7 & 52.5 & 21.1 & 30.5 & 73.4 & 140.6 \\
\hline 230 & 95 & 4 & 23 & 18 & 10 & 41.82 & 34.663 & 135.012 & 10.28 & 3.0 & 119.0 & 37.0 & 38.6 & 356.5 & 68.0 & 120.5 \\
\hline 231 & 95 & 4 & 25 & 14 & 28 & 38.31 & 34.581 & 134.992 & 14.73 & 2.3 & 251.0 & 56.2 & 177.3 & 342.6 & 87.7 & 33.8 \\
\hline 232 & 95 & 4 & 26 & 0 & 49 & 0.62 & 34.522 & 134.880 & 4.96 & 3.0 & 335.7 & 37.0 & 11.6 & 236.4 & 83.1 & 126.5 \\
\hline 233 & 95 & 4 & 27 & 12 & 54 & 22.52 & 34.544 & 134.905 & 11.91 & 2.6 & 168.3 & 68.3 & -20.6 & 266.2 & 71.0 & -157.0 \\
\hline 234 & 95 & 4 & 30 & 23 & 12 & 39.38 & 34.558 & 134.967 & 9.56 & 2.6 & 229.7 & 83.8 & 174.8 & 320.3 & 84.8 & 6.2 \\
\hline 235 & 95 & 5 & 4 & 3 & 7 & 17.89 & 34.519 & 134.868 & 3.34 & 3.3 & 140.8 & 46.6 & -12.1 & 239.2 & 81.2 & -135.9 \\
\hline 236 & 95 & 5 & 4 & 3 & 8 & 53.99 & 34.521 & 134.866 & 2.86 & 2.4 & 330.2 & 64.6 & -4.9 & 62.3 & 85.5 & -154.5 \\
\hline 237 & 95 & 5 & 4 & 5 & 53 & 16.70 & 34.696 & 135.197 & 9.17 & 2.7 & 147.8 & 64.8 & -6.0 & 240.3 & 84.6 & -154.7 \\
\hline 238 & 95 & 5 & 4 & 17 & 42 & 0.26 & 34.536 & 134.898 & 13.52 & 3.0 & 250.0 & 64.2 & 179.3 & 340.3 & 89.4 & 25.8 \\
\hline 239 & 95 & 5 & 5 & 17 & 49 & 57.53 & 34.487 & 134.874 & 5.62 & 3.2 & 154.3 & 44.7 & 87.6 & 337.7 & 45.4 & 92.4 \\
\hline 240 & 95 & 5 & 5 & 20 & 24 & 30.55 & 34.881 & 135.357 & 1.22 & 2.5 & 21.6 & 74.8 & -174.1 & 290.0 & 84.3 & -15.3 \\
\hline
\end{tabular}


Table 1. (continued).

\begin{tabular}{|c|c|c|c|c|c|c|c|c|c|c|c|c|c|c|c|c|c|c|}
\hline \multirow[b]{2}{*}{ No. } & \multirow[b]{2}{*}{$Y$} & \multirow[b]{2}{*}{$M$} & \multirow[b]{2}{*}{ D } & \multirow[b]{2}{*}{$\mathrm{H}$} & \multirow[b]{2}{*}{$\mathbf{M}$} & \multirow[b]{2}{*}{$\mathrm{S}$} & \multirow[b]{2}{*}{ Lat. } & \multirow[b]{2}{*}{ Lon. } & \multirow[b]{2}{*}{ Dep. } & \multirow[b]{2}{*}{ Mag. } & \multicolumn{3}{|c|}{ Nodal plane - 1} & \multicolumn{3}{|c|}{ Nodal plane - 2} & \multirow{2}{*}{$\begin{array}{l}\text { P - axis } \\
\text { Plun. Azim. }\end{array}$} & \multirow{2}{*}{$\begin{array}{c}\mathrm{T} \text { - axis } \\
\text { Plun. Azim. }\end{array}$} \\
\hline & & & & & & & & & & & Strike & Dip & Rake & Strike & Dip & Rake & & \\
\hline 241 & 95 & 5 & 6 & 1 & 9 & 14.28 & 34.881 & 135.387 & 4.55 & 3.1 & 31.0 & 56.1 & -153 & 285.2 & 67.9 & -37 & 41.3243 .9 & 7.4340 .5 \\
\hline 242 & 95 & 5 & 6 & 2 & 44 & 7.73 & 34.516 & 134.996 & 10.81 & 3.1 & 249.2 & 46.6 & 111.0 & 40.1 & 47.3 & & 0.3144 .6 & 74.9235 .9 \\
\hline 243 & 95 & 5 & 8 & 2 & 36 & 13.49 & 34.703 & 135.255 & 11.08 & 3.5 & 316.2 & 43.6 & 91.7 & 133.9 & 46.4 & 88.4 & 1.4225 .0 & $88.2 \quad 6.4$ \\
\hline 244 & 95 & 5 & 11 & 17 & 3 & 29.85 & 34.709 & 135.220 & 11.31 & 2.5 & 256.9 & 80.2 & -174.0 & 165.9 & 84.1 & -9.8 & 11.1121 .1 & 2.7211 .6 \\
\hline
\end{tabular}

Table 2. Focal mechanism solutions of the aftershocks $(\mathrm{M}>2.3)$ in the 2nd observation period.

\begin{tabular}{|c|c|c|c|c|c|c|c|c|c|c|c|c|c|c|c|c|c|c|c|c|}
\hline \multirow[b]{2}{*}{ No. } & \multirow[b]{2}{*}{$Y$} & \multirow[b]{2}{*}{$M$} & \multirow[b]{2}{*}{ D } & \multirow[b]{2}{*}{$\mathrm{H}$} & \multirow[b]{2}{*}{ M } & \multirow[b]{2}{*}{ S } & \multirow[b]{2}{*}{ Lat. } & \multirow[b]{2}{*}{ Lon. } & \multirow[b]{2}{*}{ Dep. } & \multirow[b]{2}{*}{ Mag. } & \multicolumn{3}{|c|}{ Nodal plane - 1} & \multicolumn{3}{|c|}{ Nodal plane - 2} & \multicolumn{2}{|c|}{$P$ - axis } & \multicolumn{2}{|c|}{$T$ - axis } \\
\hline & & & & & & & & & & & Strike & Dip & Rake & Strike & Dip & Rake & Plun. & Azim. & Plun. & Azim. \\
\hline 1 & $\overline{95}$ & 10 & 5 & 4 & 22 & 14.84 & 34.744 & 135.295 & 2.24 & 2.9 & 172.5 & & 43.8 & 50.6 & 58.2 & 130.3 & 4.9 & 113.3 & 56.2 & 15.9 \\
\hline 2 & 95 & 10 & 6 & 13 & 37 & 10.57 & 34.568 & 135.013 & 5.05 & 2.2 & 174.5 & 46.4 & 35.4 & 58.4 & 65.2 & 130.5 & 11.1 & 120.5 & 51.7 & 16.2 \\
\hline 3 & 95 & 10 & 11 & 11 & 56 & 54.86 & 34.853 & 135.403 & 5.57 & 2.7 & 67.1 & 49.4 & -170.8 & 331.1 & 83.0 & -40.9 & 33.1 & 280.7 & 22.0 & 25.9 \\
\hline 4 & 95 & 10 & 13 & 2 & 47 & 53.37 & 34.962 & 135.461 & 12.88 & 2.3 & 262.2 & 60.9 & -159.8 & 162.0 & 72.4 & -30.7 & 33.9 & 119.1 & 7.5 & 214.2 \\
\hline 5 & 95 & 10 & 13 & 14 & 41 & 52.87 & 34.578 & 135.031 & 12.93 & 2.5 & 52.4 & 36.6 & 104.6 & 214.4 & 54.7 & 79.4 & 9.2 & 312.0 & 77.4 & 88.1 \\
\hline 6 & 95 & 10 & 14 & 2 & 4 & 6.08 & 34.637 & 135.116 & 10.78 & 4.6 & 46.7 & 35.3 & 69.8 & 251.0 & 57.1 & 103.8 & 11.1 & 331.1 & 73.9 & 198.3 \\
\hline 7 & 95 & 10 & 14 & 2 & 5 & 16.58 & 34.641 & 135.129 & 10.90 & 3.5 & 342.3 & 41.3 & 26.6 & 231.7 & 72.8 & 128.1 & 18.8 & 294.2 & 47.7 & 182.2 \\
\hline 8 & 95 & 10 & 14 & 2 & 39 & 32.99 & 34.642 & 135.124 & 11.77 & 2.2 & 251.8 & 52.0 & 129.4 & 18.7 & 52.5 & 50.9 & 0.2 & 135.1 & 60.0 & 225.6 \\
\hline 9 & 95 & 10 & 14 & 21 & 27 & 27.21 & 34.567 & 134.985 & 8.60 & 2.5 & 73.2 & 61.4 & -24.3 & 175.5 & 68.8 & -149.1 & 36.5 & 36.5 & 4.8 & 303.0 \\
\hline 10 & 95 & 10 & 15 & 1 & 32 & 47.67 & 34.633 & 135.115 & 12.15 & 2.7 & 199.4 & 54.1 & 43.2 & 80.5 & 56.3 & 135.2 & 1.3 & 140.4 & 53.8 & 48.6 \\
\hline 11 & 95 & 10 & 22 & 1 & 1 & 28.93 & 34.852 & 135.224 & 9.77 & 2.4 & & 74.7 & -10.0 & 32.2 & 80.3 & -164.5 & 17.8 & 256.5 & 3.8 & 165.2 \\
\hline 12 & 95 & 10 & 23 & 17 & 14 & 34.78 & 34.725 & 135.228 & 6.15 & 2. & 326.4 & 55.6 & 6.5 & 232.7 & 84.7 & 145.4 & 19.5 & 284.5 & 27.7 & 183.8 \\
\hline 13 & 95 & 10 & 25 & 21 & 56 & 50.53 & 34.637 & 135.123 & 11.34 & 2. & 43.2 & 32.6 & 87.9 & 225.7 & 57.4 & 91.4 & 12.4 & 314.7 & 77.6 & 140.2 \\
\hline 14 & 95 & 11 & 9 & 20 & 29 & 52.55 & 34.454 & 134.832 & 8.84 & 3. & 258.6 & 52.5 & -146.3 & 146.5 & 63.9 & -42.7 & 47.9 & 107.3 & 6.9 & 204.9 \\
\hline 15 & 95 & 11 & 13 & 10 & 56 & -0.27 & 34.570 & 134.987 & 7.11 & & 25.4 & 48.9 & -174.4 & 31.7 & 85.7 & -41.2 & 31.1 & 80.1 & 24.4 & 186.0 \\
\hline 16 & 95 & 11 & 13 & 15 & 30 & 57.14 & 34.638 & 135.125 & 11.71 & 2. & 59.5 & 33.6 & 118.1 & 6.9 & 60.8 & 72.7 & 14.1 & 309.4 & 69.1 & 80.7 \\
\hline 17 & 95 & 11 & 15 & 11 & 55 & 15.77 & 34.641 & 135.101 & 10.89 & 2. & 73.2 & 50.7 & 130.2 & 200.0 & 53.8 & 51.7 & 1.7 & 316.0 & 59.9 & 49.0 \\
\hline 18 & 95 & 11 & 15 & 19 & 58 & 46.50 & 34.813 & 135.369 & 9.5 & 2. & 222.9 & 40.5 & 157.4 & 330.4 & 75.5 & 51.7 & 21.2 & 88.4 & 45.6 & 201.7 \\
\hline 19 & 95 & 11 & 16 & 16 & 23 & 57.24 & 34.536 & 134.946 & 12.38 & 2. & 202.8 & 40.4 & 103.4 & 5.5 & 50.9 & 78.9 & 5.4 & 103.3 & 79.8 & 224.8 \\
\hline 20 & 95 & 11 & 17 & 17 & 46 & 52.13 & 34.642 & 135.124 & 11.53 & 2.2 & 24.7 & 45.8 & 33.2 & 270.2 & 66.9 & 130.7 & 12.5 & 332.0 & 50.4 & 226.5 \\
\hline 21 & 95 & 11 & 19 & 15 & 42 & 19.86 & 34.528 & 134.927 & 14.54 & 2.6 & 234.3 & 73.8 & -171.6 & 142.0 & 82.0 & -16.4 & 17.2 & 97.2 & 5.7 & 189.0 \\
\hline 22 & 95 & 11 & 23 & 17 & 1 & 28.03 & 34.584 & 135.054 & 8.20 & 2.3 & 149.8 & 40.0 & 103.5 & 312.4 & 51.3 & 78.9 & 5.8 & 50.3 & 79.6 & 173.6 \\
\hline 23 & 95 & 12 & 8 & 17 & 15 & 8.57 & 34.718 & 135.228 & 11.50 & 2.7 & 175.3 & 41.4 & 26.8 & 64.6 & 72.6 & 128.2 & 18.6 & 127.1 & 47.9 & 15.2 \\
\hline 24 & 95 & 12 & 16 & 4 & 49 & 38.44 & 34.375 & 134.889 & 13.44 & 3. & 95.0 & 29.7 & 18.4 & 348.9 & 81.0 & 118.4 & 30.2 & 56.0 & 46.5 & 288.1 \\
\hline 25 & 95 & 12 & 22 & 19 & 7 & 12.05 & 34.461 & 134.854 & 9.16 & 3. & 147.6 & 41.6 & 41.2 & 24.4 & 64.1 & 123.8 & 12.7 & 90.7 & 56.9 & 340.5 \\
\hline 26 & 95 & 12 & 27 & 9 & 18 & 15.17 & 34.439 & 134.799 & 6.17 & 3. & 145.2 & 68.8 & 15.1 & 49.7 & 75.9 & 158.1 & 4.8 & 98.5 & 25.3 & 6.2 \\
\hline 27 & 96 & 1 & 3 & 20 & 55 & 30.24 & 34.905 & 135.382 & 0.69 & 3. & 294.1 & 75.7 & -11.4 & 26.9 & 78.9 & -165.5 & 18.1 & 250.9 & 2.2 & 160.2 \\
\hline 28 & 96 & 1 & 4 & 15 & 19 & 56.56 & 34.795 & 135.323 & 13.60 & 2.3 & 111.1 & 45.5 & -77.9 & 274.0 & 45.8 & -102.1 & 81.4 & 103.3 & 0.1 & 12.5 \\
\hline 29 & 96 & 1 & 5 & 19 & 29 & 14.35 & 34.698 & 135.278 & 13.29 & 2.3 & & 45.7 & & 324.1 & 57.4 & 56.1 & 6.5 & 77.5 & 61.1 & 179.4 \\
\hline 30 & 96 & 1 & 8 & 3 & 37 & 14.79 & & 135.387 & & & & & 0.5 & 281.9 & 86.9 & 25.7 & 15.5 & 53.3 & 20.2 & 149.2 \\
\hline 31 & 96 & 1 & 10 & 15 & 57 & 24.12 & & 134.972 & 14.34 & 2.2 & & & 81.6 & 76.9 & 47.3 & 97.8 & 2.0 & 161.4 & 83.9 & 51.8 \\
\hline 32 & 96 & 1 & 11 & 2 & 5 & 29.74 & 34.903 & 135.387 & 0.8 & 2. & & 70.9 & -17.8 & 213.2 & 73.3 & -160.0 & 25.8 & 75.6 & 1.6 & 344.8 \\
\hline 33 & 96 & 1 & 11 & 2 & 22 & 12.65 & 34.599 & 135.226 & 7.6 & 2. & 193.7 & 39.3 & 107.5 & 351.6 & 52.9 & 76.2 & 6.9 & 91.4 & 77.0 & 213.1 \\
\hline 34 & 96 & 1 & 15 & 7 & 20 & 24.48 & 34.537 & 134.895 & 9.95 & 2.4 & 215.9 & 54.8 & 141.5 & 330.5 & 59.4 & 42.0 & 2.7 & 92.3 & 50.1 & 185.5 \\
\hline
\end{tabular}

\section{P wave velocity}

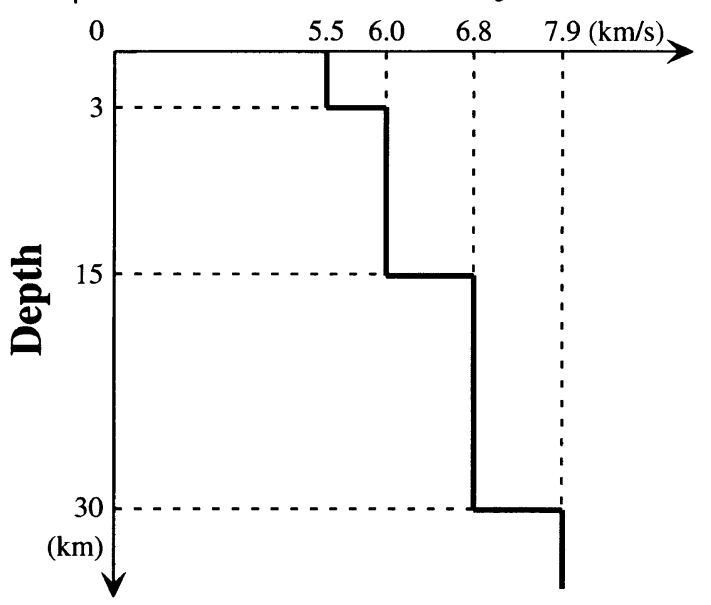

Fig. 4. P-wave velocity structure used for hypocenter determination.

\section{Data and Method}

We relocate the aftershocks with magnitudes greater than 2.3 and determine their focal mechanisms using the data obtained by GROUPS-95. It is hard to determine focal mechanisms of smaller events due to the small number of recorded seismograms and unclear initial motions of P-waves.

GROUPS-95 deployed 27 temporary seismic stations, mainly in the aftershock region, in addition to the permanent regional seismic networks: the Abuyama, Tottori and Tokushima networks operated by the Disaster Prevention Research Institute (DPRI), Kyoto University; and the Wakayama network operated by the Earthquake Research Institute (ERI), University of Tokyo. Temporary stations were operated during two periods; one from January 26, 1995 to May 12, 1995, and the other from October 1, 1995 to January 12,1996 . The installations of the 27 temporary seismic stations were completed on February 14, 1995. We note, however, that the number of the temporary seismic stations was reduced to 10 in April, 1995. For the GROUPS-95 data set, arrival times of $\mathrm{P}$ - and $\mathrm{S}$-waves and $\mathrm{P}$-wave polarities were scanned manually after several automatic data processings. However, P-wave polarities and travel times may have 
large errors, in paticular, for those stations far away from the aftershock area, because a large amount of data was processed quickly in a short time. We therefore reexamined all the P-wave arrival times and polarities for the aftershocks (M $\geq 2.3$ ) manually using the WIN system (Urabe and Tsukada, 1991). The hypocenters are determined from those re-picked arrival times by using the software HYPOMH (Hirata and Matsu'ura, 1987). The P-wave velocity structure used for the hypocenter determination is shown in Fig. 4. To improve the accuracy of the hypocenter determination, we did not use the data at the stations with epicentral distances greater than $50 \mathrm{~km}$. Further, we discarded data with unclear and/or weak onsets of P-waves due to a low signal-to-noise ratio. From the $\mathrm{P}$-wave polarities rechecked in this study, we determined the focal mechanisms for all the aftershocks with $\mathrm{M} \geq 2.3$, using the scheme of Maeda (1992). Finally, we obtained the focal mechanisms of 244 and 34 events along the mainshock rupture zone for the first and second periods, respectively. The $95 \%$ confidence limits for the obtained P- and T-axis directions are typically $\pm 5^{\circ}$ for azimuth and $\pm 15^{\circ}$ for plunge.

\section{Result}

Figures 5, 6 and 7 show the locations, focal mechanisms and directions of P-axes of the aftershocks analyzed in this study. Fault parameters of these aftershocks are listed in Table 1 (1st period) and Table 2 (2nd period). Following Katao et al. (1997), we classify the focal mechanisms into four types: normal fault with a plunge of the $\mathrm{P}$-axis $>60^{\circ}$; reverse fault with a plunge of the T-axis $>60^{\circ}$; strike-slip fault with a plunge of the Null-axis $>45^{\circ}$; and intermediate type otherwise.

On the basis of the rupture propagation of the mainshock (Ide and Takeo, 1997; Bouchon et al., 1998) and the aftershock activity (Katao et al., 1997), we divide the aftershock zone into three regions: the Kobe region (from the Akashi Strait to the Arima-Takatsuki Tectonic Line), the Awaji region in the northern part of Awaji Island, and the Akashi Strait region where the occurrence of aftershocks was less frequent than in the other two regions. We define a cluster of aftershocks based on the spatial concentration of their locations and common $\mathrm{P}$-axis orientations.

\subsection{Kobe region}

In the Kobe region (Fig. 5), many aftershocks are observed along the Rokko Fault System (Fig. 1). There are three clusters of aftershocks with strike-slip focal mechanisms near the northeastern end of the Rokko Fault, the Gosukebashi Fault and the Ashiya Fault (area a in Fig. 5). P-axis directions of these aftershocks are approximately horizontal in an ESEWNW direction.

A cluster of reverse fault type aftershocks exists near the southern end of the Gosukebashi Fault and the Otsuki Fault (area b in Fig. 5). They also have P-axes in approximately horizontal E-W or ESE-WNW directions. The focal depths of these aftershocks are 2-7 km. In contrast, most of strikeslip type in the Kobe region are located deeper than 6-8 km.

In the area from the Suwayama Fault and the Nunobiki Fault to the Suma Fault, aftershocks are mainly of strike-slip type. The majority of the P-axes of these events is in an EW or ESE-WNW direction. However, those of reverse fault type, located in a cluster along the Suma Fault at depths of about $12 \mathrm{~km}$, have P-axes in a SE-NW direction, which is almost perpendicular to the strike of the mainshock rupture, as shown in area c of Fig. 5. Most of these events occurred in the second observation period.

\subsection{Awaji region}

The occurrence of aftershocks is high in the northern half of Awaji Island (Fig. 6). Although surface rupture was observed on the Nojima Fault in the northwestern part of Awaji Island (Nakata et al., 1995; Awata et al., 1996), most of the aftershocks did not occur directly beneath the Nojima Fault, but along the Higashiura Fault and the Kariya Fault, and around the southern end of the Nojima Fault.

We recognize an aggregation of aftershocks around the southern end of the Nojima Fault (area a in Fig. 6). The azimuths of P-axes of these events are approximately in an E-W or ESE-WNW direction, which are the same as those for most aftershocks in the Kobe region. However, the aftershocks in this area are distributed in a wide range of depths, locations, and are of various focal mechanism types. We consider that this aggregation of aftershocks can not be defined as cluster.

There are many aftershocks in the northeastern part of Awaji Island. These events are not in clusters like those in the Kobe region. Their directions of P-axes are also highly variable. Synthetic tests show that this is not caused by the uncertainty of focal mechanism determination. Many reverse fault type events occurred here, compared to those in the Kobe region. Mechanism types of aftershocks do not show any coherent patterns in a small area.

\subsection{Akashi Strait}

The aftershocks activity in the Akashi Strait (Fig. 7) is extremely low, as reported by Hirata et al. (1996) and Nakamura and Ando (1996). Four foreshocks occurred in this area, together with the mainshock. We find two small clusters in this area. One is located at a depth of about $15 \mathrm{~km}$ beneath the central part of the Akashi Strait (area a in Fig. 7). The aftershocks in this cluster are mainly of strike-slip type with $\mathrm{P}$-axes rotated clockwise about $10^{\circ}$ relative to those of the mainshock and the two foreshocks determined by Katao et al. (1997).

The other cluster is located at a depth of about $6 \mathrm{~km}$ beneath the eastern part of the Akashi Strait (area b in Fig. 7). Most of the events in this cluster are of reverse fault type. Paxes of these aftershocks are oriented approximately in N-S, in contrast with the regional compressional direction of $\mathrm{E}-\mathrm{W}$ to ESE-WNW.

\subsection{Temporal variation in aftershock mechanisms}

The temporal variation in $\mathrm{P}$-axes directions related to a large earthquake was studied by Zhao et al. (1997). They reported that the average direction of $\mathrm{P}$-axes in the epicentral area of the 1994 Northridge earthquake changed from $\mathrm{N} 30^{\circ} \mathrm{E}$ to $\mathrm{N} 13^{\circ} \mathrm{E}$ before and after the mainshock, suggesting the coseismic stress rotation. Furthermore, they showed that the stress orientation rotated back to its original orientation $\left(\mathrm{N} 34^{\circ} \mathrm{E}\right)$ during the two years following the mainshock.

Here, we consider whether or not the P-axis directions of aftershocks show any temporal changes for the Hyogo-ken Nanbu earthquake. Because P-axis directions vary widely over the aftershock region (Fig. 5, 6, and 7), we investigate the possibility of a temporal change in $\mathrm{P}$-axis directions sep- 


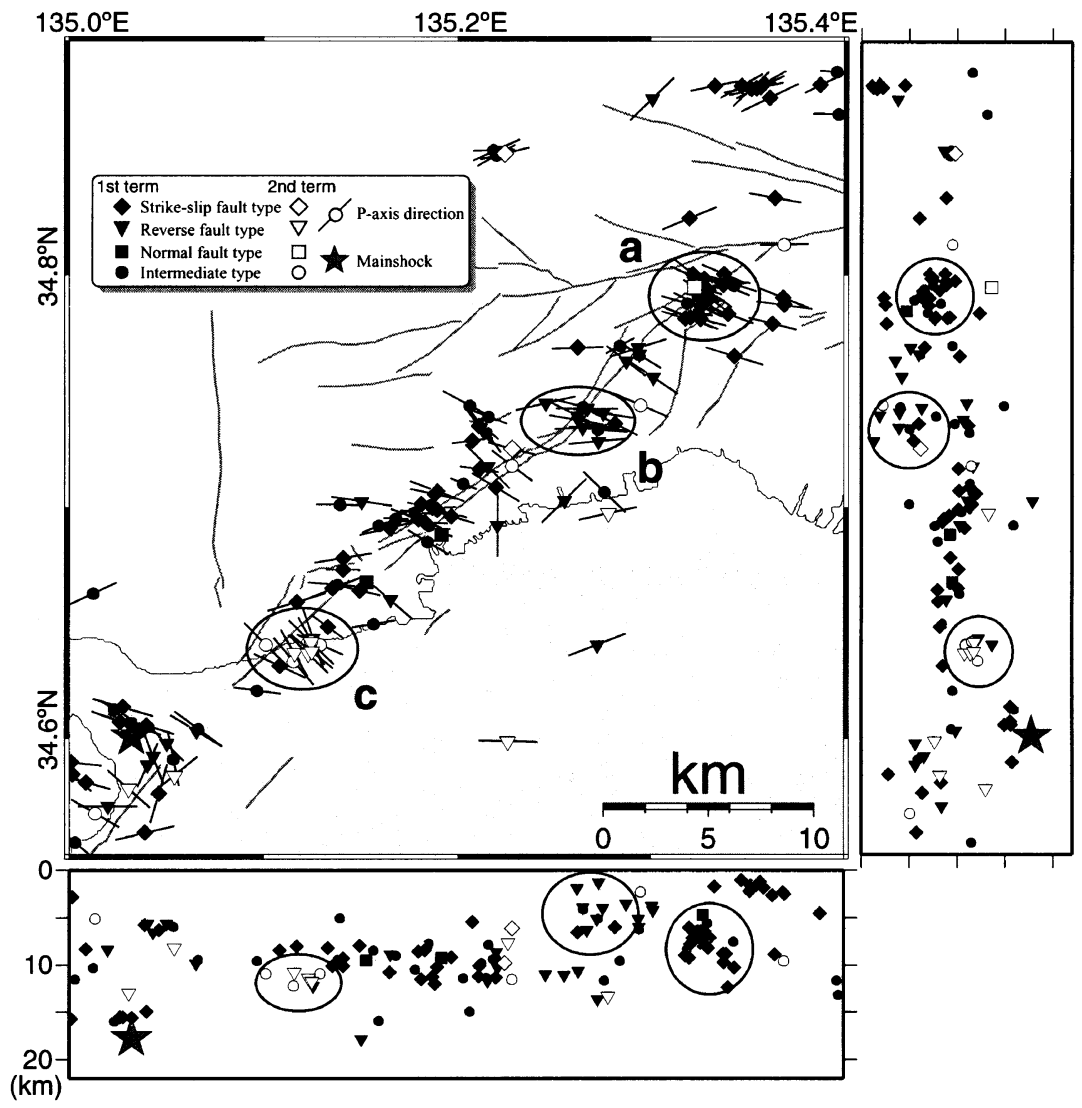

Fig. 5. Type of focal mechanisms of aftershocks in the Kobe region. Diamonds represent strike-slip type, triangles for reverse-fault type and squares for normal-fault type. Circles show intermediate types. Solid and open symbols represent the aftershocks observed in the 1st (from Jan. 26 to May 12 , 1995) and 2nd observation periods (from Oct. 1, 1995 to Jan. 12, 1996), respectively. Short bars attached to epicenter circles represent the horizontal projections of P-axes. Star shows the hypocenter of the mainshock.

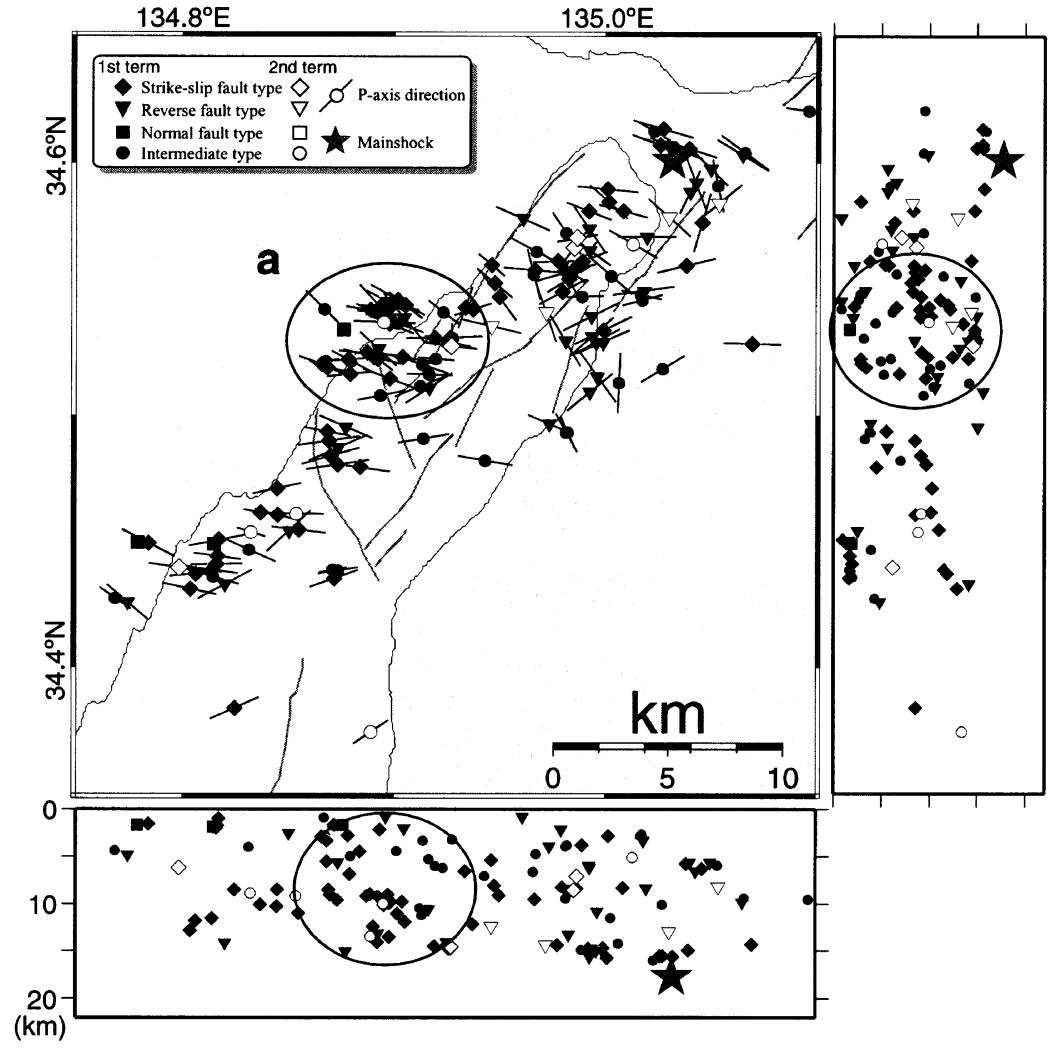

Fig. 6. Type of focal mechanisms of aftershocks in the Awaji region. All symbols are the same as those in Fig. 5. 


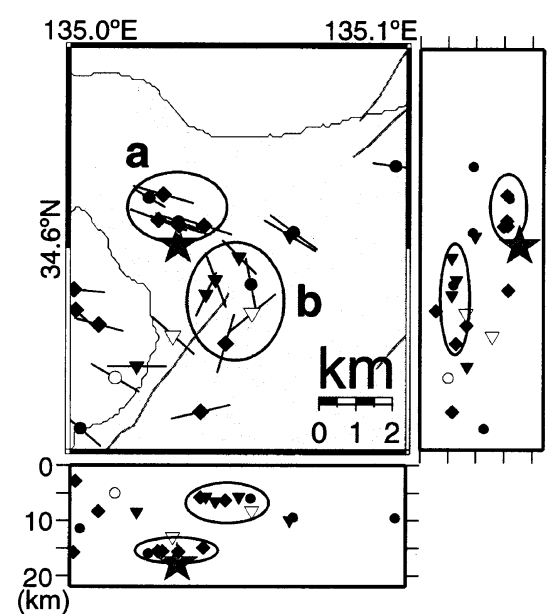

Fig. 7. Type of focal mechanisms of aftershocks in Akashi Strait. All symbols are the same as those in Fig. 5.

arately for each cluster that we identified. We use the focal mechanism solutions from January 17, 1995 to January 28, 1995 determined by Katao et al. (1997), in addition to those determined in this study. Within the accuracy of the present data set, we observe no temporal changes in focal mechanism orientations in area $\mathrm{c}$ in Fig. 5 from the first to the second period of observation (Fig. 8).

\section{Discussion}

Most aftershocks $(M \geq 2.3)$ have strike-slip or reverse fault mechanisms with P-axes approximately in an E-W or ESE-WNW direction (Fig. 5, 6, and 7). Katao et al. (1997) reported that $\mathrm{P}$-axes of many aftershocks are approximately in an E-W direction. They compared their results with the regional stress field in the Kinki District: the E-W compression before the Hyogo-ken Nanbu earthquake reported by Iio (1996). They suggested that the mainshock rupture zone was still controlled by the regional stress field of E-W compression even after the large mainshock. In this study, events with $\mathrm{P}$-axes in E-W directions are observed along the mainshock rupture zone for aftershocks within the clusters of the strike-slip events close to the Rokko Fault and the northern end of the Gosukebashi Fault (area a in Fig. 5), the cluster of reverse fault events near the southern end of the Gosukebashi Fault (area b in Fig. 5) and the aggregation of aftershocks near the southern edge of the Nojima Fault (area a in Fig. 6). We consider that these regions are controlled by the regional stress field of approximately E-W compression after the mainshock.

On the other hand, we observe many aftershocks whose Paxes are inconsistent with the regional stress. A small number of normal mechanism events occurred along the mainshock rupture zone, as Katao et al. (1997) pointed out. The aftershocks along the Suma Fault show mainly reverse mechanism solutions with P-axes in a SE-NW direction (area c in Fig. 5). In the eastern part of the Akashi Strait (area b in Fig. 7), the aftershocks with N-S compressional focal mechanism form a cluster. We further detect a large scatter of Paxis directions for the aftershocks in the northeastern part of Awaji Island (Fig. 6). Such a complex pattern of aftershock focal mechanisms was also observed in the case of the 1989

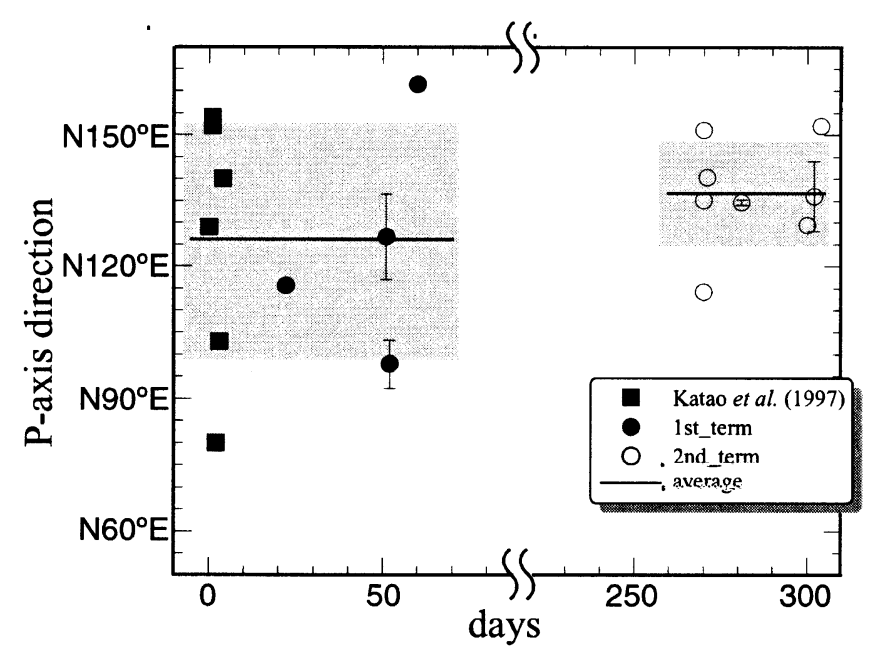

Fig. 8. Temporal variations in P-axis directions for the cluster of aftershocks along the Suma Fault (area c shown in Fig. 5). Solid and open circles represent the $\mathrm{P}$-axis direction of those observed in the 1 st and 2 nd observation periods, respectively. Solid squares for the first 10 days after the mainshock are data determined by Katao et al. (1997). Error bars indicate a range of possible focal solutions.

Loma Prieta earthquake (Michael et al., 1990; Oppenheimer, 1990).

In this study, we call aftershocks that are inconsistent with the regional stress field "atypical." That is, atypical aftershocks are defined as those with a P-axis oriented more than $45^{\circ}$ from the E-W direction or with a $\mathrm{P}$-axis plunge of more than $45^{\circ}$. The 48 atypical aftershocks identified in the present data set correspond to about $17 \%$ of the total. We compare the spatial distribution of the aftershocks, especially the atypical aftershocks (Fig. 9), with the inverted slip and stress distributions for the mainshock and other parameters to clarify the cause of the occurrence of the atypical aftershocks. The Coulomb stress change by the mainshock was calculated and compared with the induced seismicity rate change in the surrounding areas (Hashimoto, 1997; Toda et al. 1998). However, the aftershocks discussed in this study occurred on the mainshock slip plane or very close to it. Because the present precision of hypocenter determination and slip models are not enough to determine whether each aftershock occurs on the hangingwall or footwall side of the mainshock slip plane. It is difficult to explain the atypical focal mechanisms using the Coulomb stress change.

Figure 10 shows a cross section of the hypocenter distribution of the aftershocks relocated in this study with contours of the final stress along the mainshock rupture zone, inferred by Bouchon et al. (1998). Bouchon et al. (1998) determined the stress and the slip distributions using an inversion technique from strong motion data. In the Kobe region, aftershocks are observed in a belt-like area at depths of about 10 $\mathrm{km}$. Higher final stress is also distributed in the depth range from about $10 \mathrm{~km}$ to $15 \mathrm{~km}$. In the Awaji region, aftershocks are observed over a wide depth range, and higher final stress is also distributed widely, in the depth from the surface to 15 $\mathrm{km}$. The distribution of aftershocks corresponds to the areas with higher final stress in the mainshock rupture zone. The shape of the aftershock distribution appears to depend on the degree of the final stress. 


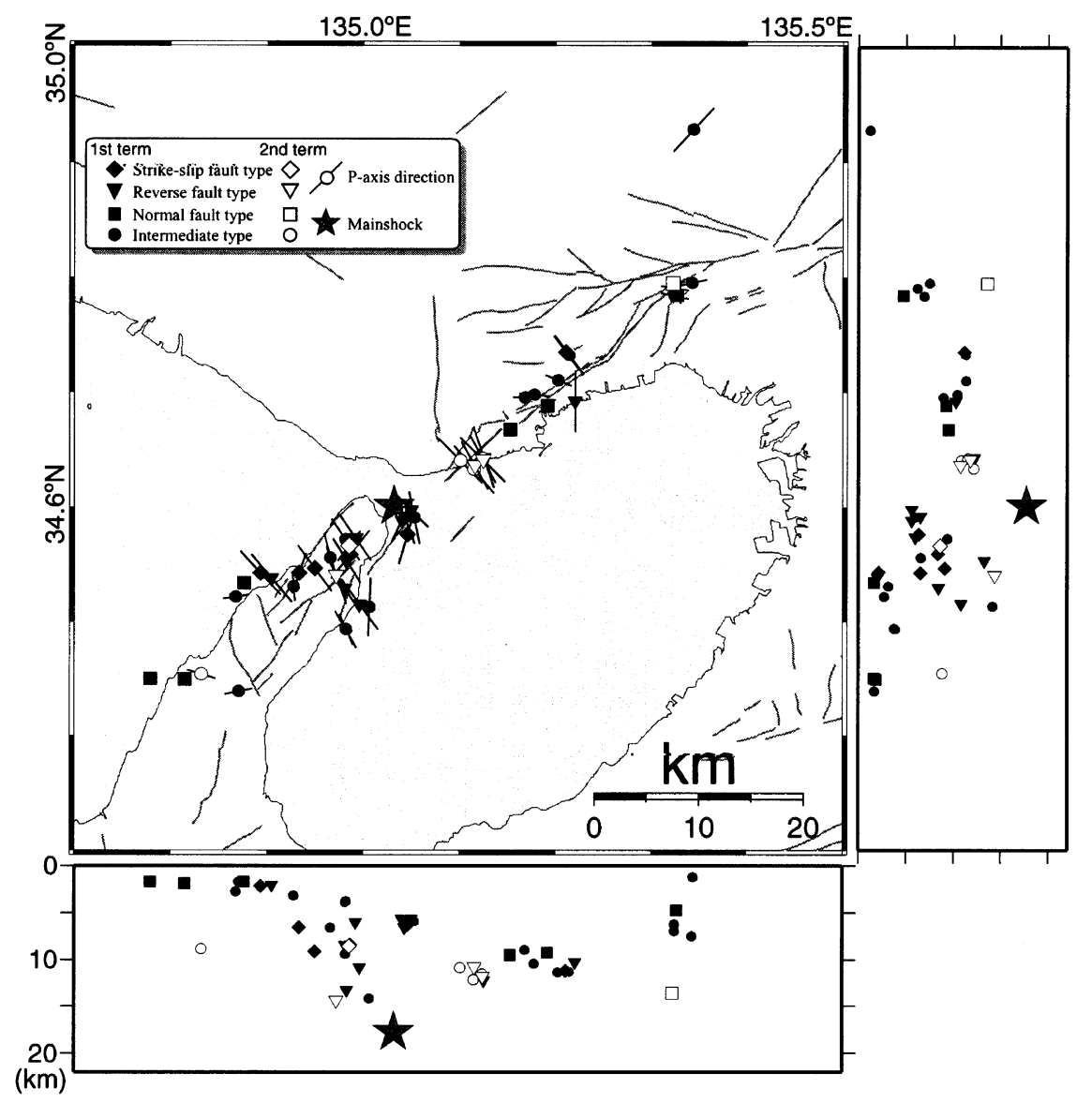

Fig. 9. Distribution of atypical aftershocks with the direction of P-axis inconsistent with the regional stress. All symbols are the same as those in Fig. 5.

Figure 11 shows the locations of the atypical aftershocks and the distribution of the final stress. In the Kobe region, there are atypical aftershocks around the Suwayama Fault and along the Suma Fault (Fig. 9). The atypical aftershocks are located at the boundary between the areas of higher and lower final stress. The locations of the atypical aftershocks are compared with the distribution of the slip for the mainshock shown in Fig. 12. In the Kobe region, the slip of the mainshock is the greatest below about $10 \mathrm{~km}$. For the atypical aftershocks around the Suwayama Fault, especially the events of normal or close to normal fault type near the boundary between the Suwayama Fault and the Suma Fault, we suppose that they are caused by the local stress field perturbed by the difference of displacements at the depth of 10 $\mathrm{km}$. From the comparison with the stress and the slip distributions for the cluster of mainly reverse fault type events observed along the Suma Fault, we can see that these are located on the edge of high stress or large slip area. The focal mechanisms of aftershocks in this cluster are similar to each other (there are two types, reverse and intermediate types, because of the classification of mechanisms), and they are aligned parallel to the active fault at almost the same depths. These are different from other atypical aftershocks distributed in the shallower part of the cluster of strike-slip events in the Kobe region. P-axis directions of these aftershocks are perpendicular to the strike of the active fault (Fig. 9). We consider two possible explanations for this cluster of the atypical aftershocks of mainly reverse fault type along the Suma Fault. One explanation is that the events are caused by the local stress field disturbed by the heterogeneous displacement near the boundaries of the mainshock rupture. The other is that the shear stress from the regional stress field was adequately canceled by the mainshock slip. Along the San Andreas fault system in central California show the NE-SW compression that is nearly perpendicular to the strike of the fault (Zoback et al., 1987). In the case of this study, however, the regional stress field of E-W compression was obsereved in a wide area around the rupture faults before/after the mainshock. We consider that the anomalous Paxis directions nearly perpendicular to the strike of the fault are caused by the heterogeneous coseismic slip.

In the Awaji region, there are many atypical aftershocks in the northeastern part of Awaji Island, where P-axis directions of the aftershocks show a large scatter (Fig. 9). The mainshock slip is large in the shallower part of this region. The large slip area extends to a depth of about $15 \mathrm{~km}$ (Fig. 12). Around the northern end of Awaji Island, however, there is an area of lower final stress where the mainshock slip was also very small. The atypical aftershocks in the Awaji region are located at this boundary of the rupture following the mainshock slip (in Fig. 12, the aftershocks plotted on the northeastern side of the large slip area, and in Fig. 9, aftershocks located around the Higashiura Fault). The heterogeneity of the mainshock slip altered the local stress field of this area. Moreover, in the northeastern part of Awaji Island, the contour intervals of the slips in the region where atyp- 


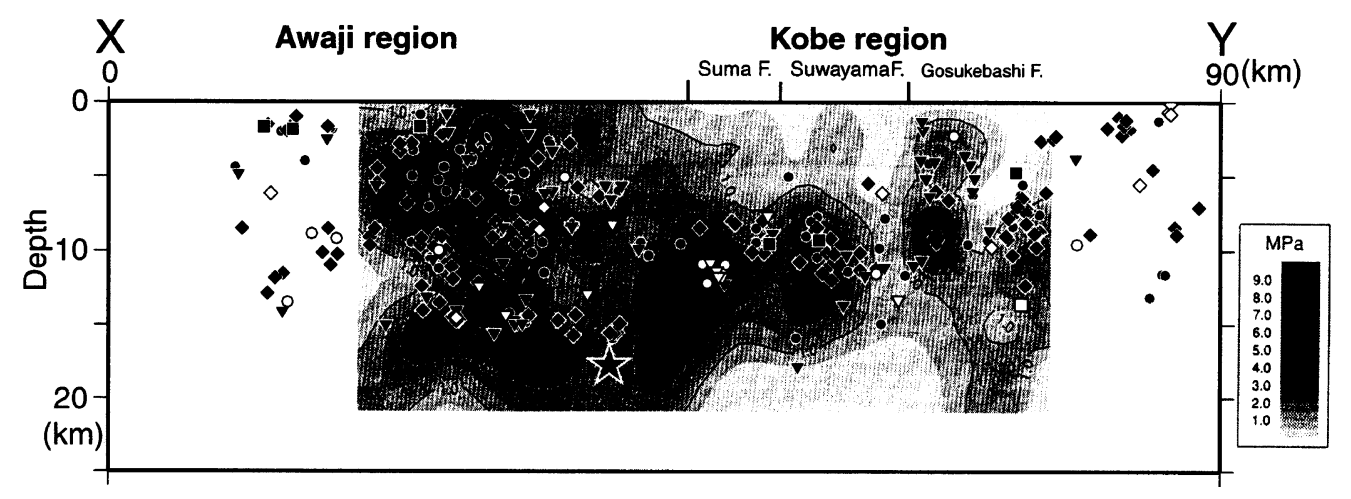

Fig. 10. Cross section of the hypocenter distribution of the aftershocks with the final stress on the mainshock faults of Bouchon et al. (1998), along the $\mathrm{X}-\mathrm{Y}$ line of Fig. 1. All symbols are the same as those in Fig. 5. The contour interval of final stress is $1.0 \mathrm{Mpa}$.

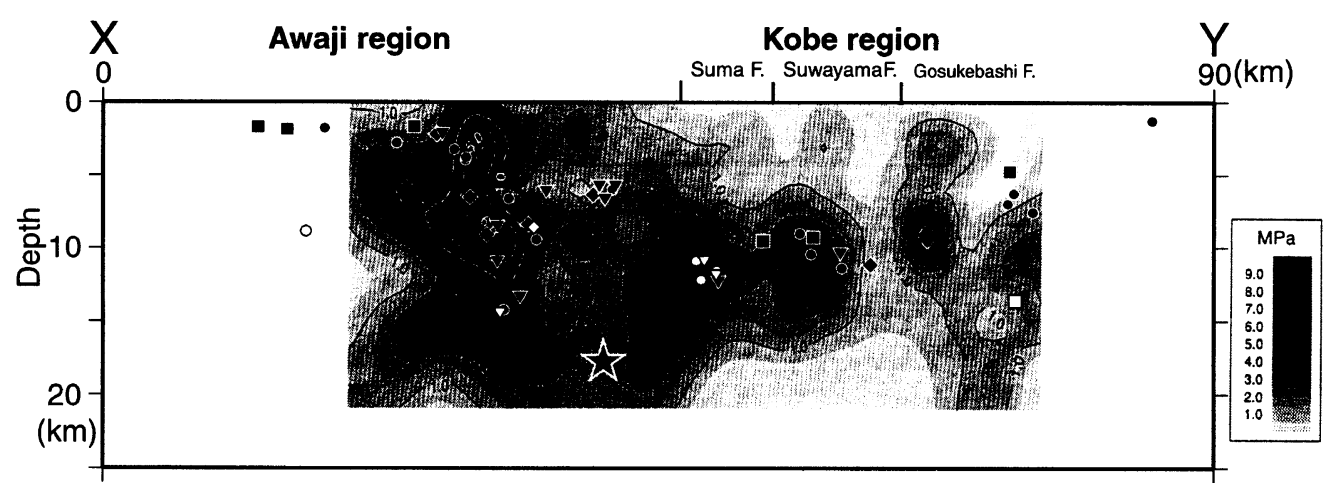

Fig. 11. Cross section of the locations of the atypical aftershocks with the final stress on the mainshock faults of Bouchon et al. (1998), along the X-Y line of Fig. 1. All symbols are the same as those in Fig. 5. The contour interval of final stress is $1.0 \mathrm{MPa}$.

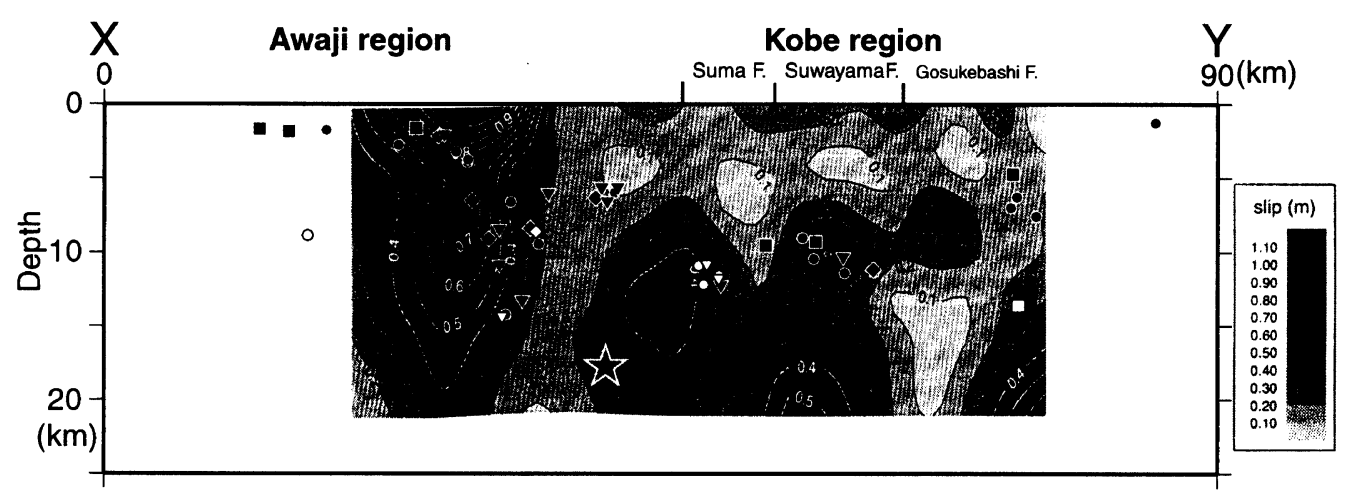

Fig. 12. Cross section of the locations of the atypical aftershocks with the coseismic slip distribution of Bouchon et al. (1998), along the X-Y line of Fig. 1. All symbols are the same as those in Fig. 5. The contour interval of slip is $0.1 \mathrm{~m}$.

ical aftershocks occurred are closer than those in the Kobe region, as shown in Fig. 12. That is, the heterogeneity of the mainshock slip is greater on Awaji Island, and the stress field is disturbed to a higher degree, causing atypical aftershocks there.

Tadokoro et al. (1999) analyzed S-wave splitting in and around the aftershock region. They showed that the polarization directions of the faster shear wave $(\psi)$ in the northern part of Awaji Island were various to strikes of the known active faults. Their results seem to agree with the P-axis directions of the aftershocks determined in this study. For the region where $\psi$ is not consistent with the regional stress field, the inconsistency appears to be caused by its compli- cated geology in the northeastern part of Awaji Island. These observations may be partly originated from complicated rupture of the mainshock.

For the cluster of the aftershocks with P-axis in the N-S direction at depths of about $6 \mathrm{~km}$ in the eastern part of Akashi Strait (area b in Fig. 7), there is no evidence of the complication in the distribution of the mainshock slip or final stress field. Zhao and Negishi (1998) determined P- and S-wave velocity structures and the distribution of Poisson's ratio in the aftershock region (Fig. 13). There is an area of slow Swave velocity and high Poisson's ratio in a shallower part of Akashi Strait. This cluster of aftershocks with compressional focal mechanisms might correspond to the boundary 
(a)

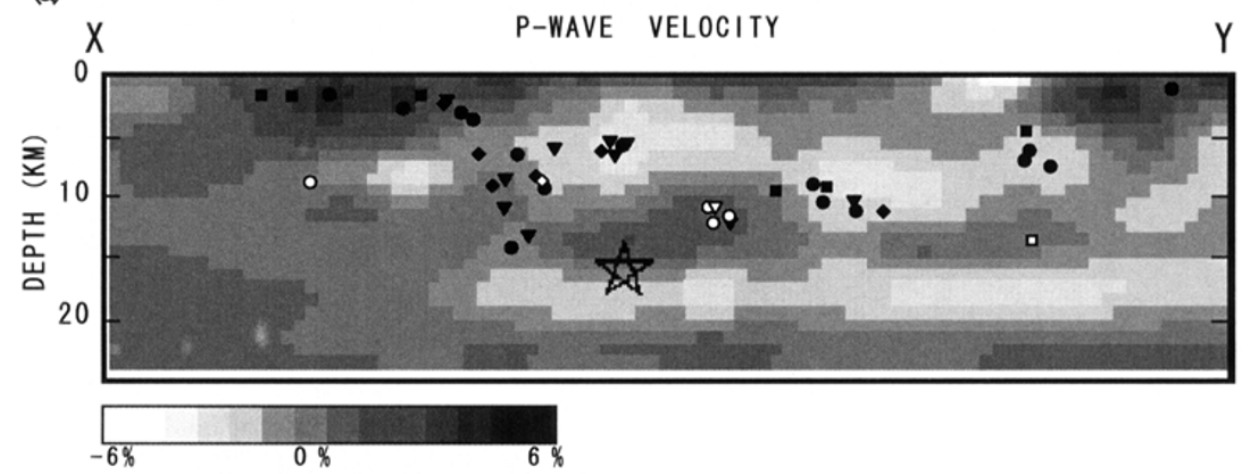

(b)
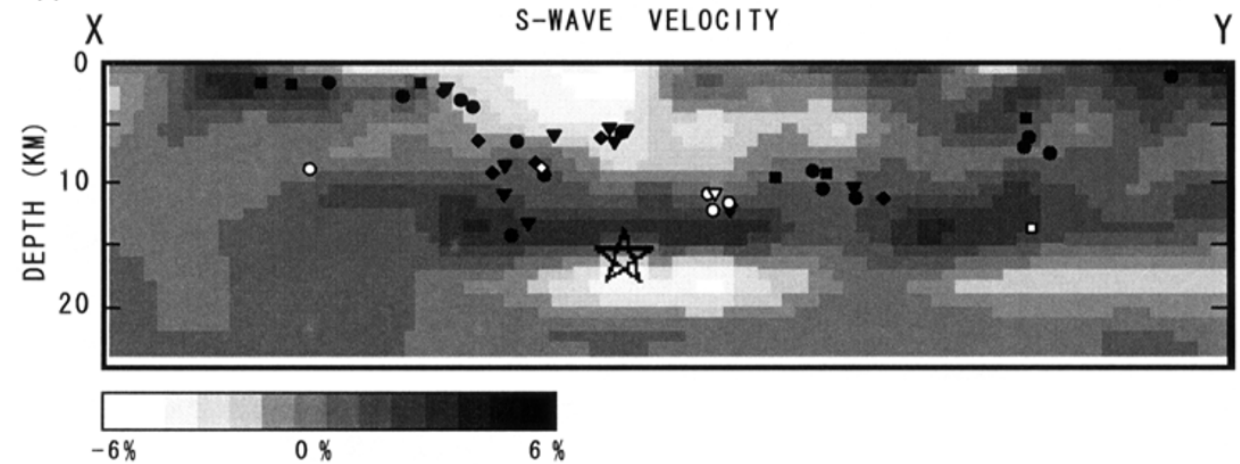

(c)

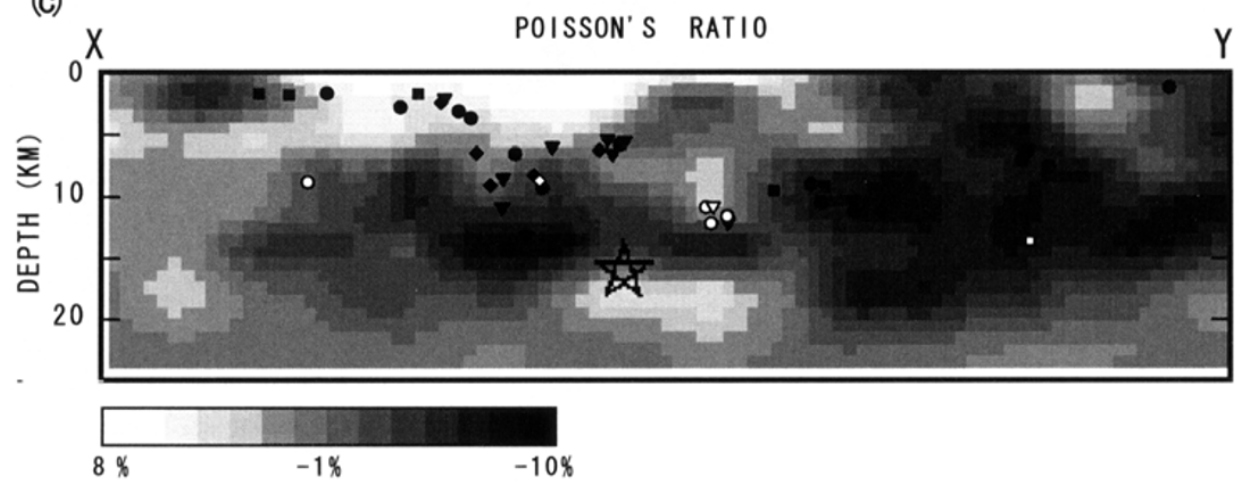

Fig. 13. Cross section of the locations of the atypical aftershocks in comparison with the (A) P-wave velocity, (B) S-wave velocity and (C) Poisson's ratio from Zhao and Negishi (1998), along the X-Y line of Fig. 1. All symbols are the same as those in Fig. 5.

at which S-wave velocity changes rapidly. From the velocity structure of the crust only, however, we cannot surmise the cause of the atypical aftershocks with P-axes in a N-S direction, which is perpendicular to the regional stress field. We cannot detect any obvious corresponding anomaly in Pwave velocity structure. A possible cause of the occurrence of these aftershocks is the geometrical gap in the fault segment beneath the Akashi Strait (Nakamura and Ando, 1996; Takahashi et al., 1996), and the resulting perturbation of the stress field.

\section{Conclusions}

We relocate the hypocenters of the aftershocks of the 1995 Hyogo-ken Nanbu earthquake and determine their focal mechanisms using seismic records obtained by GROUPS95. The focal mechanisms of the aftershocks are mainly consistent with the regional stress field, which shows E-W or ESE-WNW compression. However, there are groups of events with different $\mathrm{P}$-axis directions. In the Kobe region, there are clusters of aftershocks which have almost the same direction of P-axes and type of focal mechanisms. In the northeastern part of Awaji Island, P-axis directions of aftershocks are widely spreaded. In Akashi Strait, there is a cluster of events with N-S compression, almost perpendicular to the regional stress field. We compare the locations of such "atypical" aftershocks with the coseismic slip distribution, the stress distribution after the mainshock, and other geophysical parameters. The atypical aftershocks are mainly located near the boundaries between high and low stress areas, and between slipped and unslipped zones. We suggest that the atypical aftershocks in the mainshock rupture zone are caused by the disturbance of the local stress field due to the heterogeneities of the distributions of the final stress and the mainshock. 
Acknowledgments. We thank Dapeng Zhao and Hiroaki Negishi for providing their diagram of seismic wave velocity structure. We thank Keiichi Tadokoro for his help to obtain the aftershock data. All participants in GROUPS-95 enable us to do this study. We are grateful to Prof. James Mori and Dr. Peiliang Xu for their careful reading and correction of our manuscript. Comments of Dr. Shigeki Horiuchi, Dr. Kiyoshi Yomogida and an anonymous reviewer are useful to improve our manuscript.

\section{References}

Aki, K. and P. G. Richards, Quantitative Seismology, Vol. 1, W. H. Freeman and Company, New York, 1980.

Awata, Y., K. Mizuno, Y. Sugiyama, R. Imura, K. Shimokawa, K. Okumura, E. Tsukuda, and K. Kimura, Surface fault ruptures on the northwest coast of Awaji Island associated with the Hyogo-ken Nanbu earthquake of 1995, Japan, J. Seismol. Soc. Jpn. 2, 49, 113-124, 1996 (in Japanese with English abstract).

Bouchon, M., H. Sekiguchi, K. Irikura, and T. Iwata, Some characteristics of the stress field of the 1995 Hyogo-ken Nanbu (Kobe) earthquake, $J$. Geophys. Res., 103(B10), 24271-24282, 1998.

Hashimoto, M., Correction to "Static stress changes Associated with the Kobe Earthquake: Calculation of changes in Coulomb failure function and comparison with seismicity change", J. Seismol. Soc. Jpn. 2, 50, 21-27, 1997 (in Japanese with English abstract).

Hiramatsu, Y., N. Hayashi, M. Furumoto, and H. Katao, Temporal changes in coda $\mathrm{Q}^{-1}$ and $b$ value due to the static stress change associated with the 1995 Hyogo-ken Nanbu earthquake, J. Geophys. Res., 105(B3), 6141$6151,2000$.

Hirata, N. and M. Matsu'ura, Maximum-likelihood estimation of hypocenter with origin time eliminated usuing nonlinear inversion technique, Phys. Earth Planet. Inter., 47, 50-61, 1987.

Hirata, N., S. Ohmi, S. Sakai, K. Katsumata, S. Matsumoto, T. Takanami, A. Yamamoto, T. Iidaka, T. Urabe, M. Sekine, T. Ooida, F. Yamazaki, H. Katao, Y. Umeda, M. Nakamura, N. Seto, T. Matsushima, H. Shimizu, and Japanese University Group of the Urgent Joint Observation for the 1995 Hyogo-ken Nanbu Earthquake, Urgent joint observation of aftershocks of the 1995 Hyogo-ken Nanbu earthquake, J. Phys. Earth, 44, 317-328, 1996

Ide, S. and M. Takeo, Determination of constitutive relations of fault slip based on seismic wave analysis, J. Geophys. Res., 102(B12), 2737927391, 1997.

Iio, Y., Depth-dependent change in the focal mechanism of shallow earthquakes: Implications for brittle-plastic transition in a seismogenic region, J. Geophys. Res., 101(B5), 11209-11216, 1996.
Katao, H., N. Maeda, Y. Hiramatsu, Y. Iio, and S. Nakao, Detailed mapping of focal mechanisms in/around the 1995 Hyogo-ken Nanbu earthquake rupture zone, J. Phys. Earth, 45, 105-119, 1997.

Maeda, N., A method of determining focal mechanisms and quantifying the uncertainty of the determining focal mechanisms for microearthquakes, Bull. Seismol. Soc. Am., 82, 2410-2429, 1992.

Mendoza, C. and S. H. Hartzell, Aftershock patterns and main shock faulting, Bull. Seismol. Soc. Am., 78, 1438-1449, 1988.

Michael, A. J., W. L. Ellsworth, and D. H. Oppenheimer, Coseismic stress changes induced by the 1989 LomaPrieta, California earthquake, Geophys. Res. Lett., 17, 1441-1444, 1990.

Nakamura, M. and M. Ando, Aftershock distribution of the January 17, 1995 Hyogo-ken Nanbu earthquake determined by the JHD method, $J$. Phys. Earth, 44, 329-335, 1996.

Nakata, T., K. Yomogida, J. Odaka, T. Sakamoto, K. Asahi, and N. Chida, Surface fault ruptures associated with the 1995 Hyogo-ken Nanbu earthquake, J. Geogr., 104, 127-142, 1995 (in Japanese with English abstract).

Oppenheimer, D. H., Aftershock slip behavior of the 1989 Loma Prieta, California Earthquake, Geophys. Res. Lett., 17, 1441-1444, 1990.

Tadokoro, K., M. Ando, and Y. Umeda, S wave splitting in the aftershock region of the 1995 Hyogo-ken Nanbu earthquake, J. Geophys. Res., 104(B1), 981-991, 1999.

Takahashi, N., K. Suyehiro, M. Shinohara, A. Kubo, A. Nishizawa, and H. Matsuoka, Aftershocks and faults of the Hyogo-ken Nanbu earthquake beneath Akashi Strait, J. Phys. Earth, 44, 337-347, 1996.

Toda, S., R. S. Stein, P. A. Reasenberg, J. H. Dieterich, and A. Toshida, Stress transferred by the $1995 \mathrm{Mw}=3.9$ Kobe, Japan, shock: Effect on aftershocks and future earthquake probabilities, J. Geophys. Res., 103(B10), 24543-24565, 1998.

Urabe, T. and S. Tsukada, A workstation-assisted processing system for waveform data from microearthquake networks, Abstracts of Spring Meeting of Seismological Society of Japan, 70, 1991 (in Japanese).

Zhao, D. and H. Negishi, The 1995 Kobe earthquake: Seismic image of the source zone and its implications for the rupture nucleation, J. Geophys. Res., 103(B5), 9967-9986, 1998.

Zhao, D., H. Kanamori, and D. Wiens, State of stress before and after the 1994 Northridge earthquake, Geophys. Res. Lett., 24, 519-522, 1997.

Zoback, M. D, M. L. Zoback, V. S. Mount, J. Suppe, J. P. Eaton, J. H. Healy, D. Oppenheimer, P. Reasenberg, L. Jones, C. B. Raleigh, I. G. Wong, O. Scotti, and C. Wentworth, New evidence on the state of stress of the San Andreas fault system, Science, 238, 1105-1111, 1987.

H. Yamanaka, Y. Hiramatsu, and H. Katao (e-mail: katao@rcep.dpri. kyoto-u.ac.jp) 